\title{
Deep imaging survey of young, nearby austral stars ${ }^{\star}$ VLT/NACO near-infrared Lyot-coronographic observations
}

\author{
G. Chauvin ${ }^{1}$, A.-M. Lagrange ${ }^{1}$, M. Bonavita ${ }^{2,3}$, B. Zuckerman $^{4}$, C. Dumas ${ }^{5}$, M. S. Bessell ${ }^{6}$, J.-L. Beuzit ${ }^{1}$, \\ M. Bonnefoy ${ }^{1}$, S. Desidera ${ }^{2}$, J. Farihi ${ }^{7}$, P. Lowrance ${ }^{8}$, D. Mouillet $^{1}$, and I. Song ${ }^{9}$ \\ ${ }^{1}$ Laboratoire d'Astrophysique, Observatoire de Grenoble, UJF, CNRS, 414 rue de la piscine, 38400 Saint-Martin d'Hères, France \\ e-mail: Gael.Chauvin@obs.ujf-grenoble.fr \\ 2 INAF - Osservatorio Astronomico di Padova, Vicolo dell' Osservatorio 5, 35122 Padova, Italy \\ 3 Universita' di Padova, Dipartimento di Astronomia, Vicolo dell'Osservatorio 2, 35122 Padova, Italy \\ 4 Department of Physics \& Astronomy and Center for Astrobiology, University of California: Los Angeles, Box 951562, CA 90095, \\ USA \\ 5 European Southern Observatory: Casilla 19001, Santiago 19, Chile \\ ${ }^{6}$ Research School of Astronomy and Astrophysics Institute of Advance Studies, Australian National University: Cotter Road, \\ Weston Creek, Canberra, ACT 2611, Australia \\ 7 Department of Physics \& Astronomy, University of Leicester, Leicester LE1 7RH, UK \\ 8 Spitzer Science Center, IPAC/Caltech: MS 220-6, Pasadena, CA 91125, USA \\ 9 Department of Physics \& Astronomy, University of Georgia, Athens, GA 30602-2451, USA
}

Received 23 January 2009 / Accepted 14 June 2009

\section{ABSTRACT}

\begin{abstract}
Context. High contrast and high angular resolution imaging is the optimal search technique for substellar companions to nearby stars at physical separations larger than typically $10 \mathrm{AU}$. Two distinct populations of substellar companions, brown dwarfs and planets, can be probed and characterized. As a result, fossile traces of processes of formation and evolution can be revealed by physical and orbital properties, both for individual systems and as an ensemble.

Aims. Since November 2002, we have conducted a large, deep imaging, survey of young, nearby associations of the southern hemisphere. Our goal is detection and characterization of substellar companions with projected separations in the range 10-500 AU. We have observed a sample of 88 stars, primarily G to M dwarfs, younger than $100 \mathrm{Myr}$, and within $100 \mathrm{pc}$ of Earth.

Methods. The VLT/NACO adaptive optics instrument of the ESO Paranal Observatory was used to explore the faint circumstellar environment between typically 0.1 and $10^{\prime \prime}$. Diffraction-limited observations in $H$ and $K_{\mathrm{s}}$-band combined with Lyot-coronagraphy enabled us to reach primary star-companion brightness ratios as small as $10^{-6}$. The existence of planetary mass companions could therefore be probed. We used a standardized observing sequence to precisely measure the position and flux of all detected sources relative to their visual primary star. Repeated observations at several epochs enabled us to discriminate comoving companions from background objects.

Results. We report the discovery of 17 new close (0.1-5.0") multiple systems. HIP 108195 AB and C (F1 III-M6), HIP 84642 AB $(a \sim 14 \mathrm{AU}, \mathrm{K} 0-\mathrm{M} 5)$ and TWA22 AB $(a \sim 1.8 \mathrm{AU}$; M6-M6) are confirmed comoving systems. TWA22 AB is likely to be a rare astrometric calibrator that can be used to test evolutionary model predictions. Among our complete sample, a total of 65 targets were observed with deep coronagraphic imaging. About 240 faint companion candidates were detected around 36 stars. Follow-up observations with VLT or HST for $83 \%$ of these stars enabled us to identify a large fraction of background contaminants. Our latest results that pertain to the substellar companions to GSC 08047-00232, AB Pic and 2M1207 (confirmed during this survey and published earlier), are reviewed. Finally, a statistical analysis of our complete set of coronagraphic detection limits enables us to place constraints on the physical and orbital properties of giant planets between typically 20 and $150 \mathrm{AU}$.
\end{abstract}

Key words. instrumentation: adaptive optics - instrumentation: high angular resolution - methods: observational methods: statistical - brown dwarfs - planetary systems

\section{Introduction}

The search for substellar objects, either isolated or as companions to nearby stars, has strongly motivated observers during the past two decades. The detection and characterization of substellar objects aids in understanding the formation and evolution of stars, brown dwarfs and planets. Since the discovery of the first unambiguous brown dwarf G1229 B (Nakajima et al. 1995), the

* Table 8 is only available in electronic form at the CDS via anonymous ftp to cdsarc.u-strasbg.fr (130.79.128.5) or via http://cdsweb.u-strasbg.fr/cgi-bin/qcat?J/A+A/509/A52 development of new imaging instruments and observing techniques has diversified. Large surveys (2MASS, Skrutskie et al. 1997; DENIS, Epchtein et al. 1997; SLOAN, York et al. 2000) are the best method for the study of isolated substellar objects. Hundreds of brown dwarfs have been discovered in the field motivating the introduction of the cool new $\mathrm{L}$ and $\mathrm{T}$ spectral classes (Delfosse et al. 1997; Kirkpatrick et al 1999; Burgasser et al. 1999). Dedicated spectroscopic observations of these cool atmospheres offer an opportunity to study physical and chemical processes such as grain and molecule formation and vertical mixing and cloud coverage. In the field, in young open clusters 
and in star forming regions, study of the intial-mass function and of stellar and substellar multiplicity reveals an apparent continuous sequence supporting the idea that the same mechanisms (collapse, fragmentation, ejection, photo-evaporation of accretion envelopes) form objects over a wide range of masses from stars down to planets, as predicted by some theoretical models (Bonnell et al. 2007; Burgasser et al. 2007; Zuckerman \& Song 2009). Despite limited spatial resolution, a dozen substellar companions to nearby stars have been discovered with wide ( $\geq 100$ AU) orbits (e.g., Goldman et al. 1999; Kirkpatrick et al. 2000; Wilson et al. 2001).

To access the near ( $\leq 5 \mathrm{AU})$ environment of stars, observing techniques other than direct imaging (e.g., precision radial velocity, transit, micro-lensing, pulsar-timing), are best suited. The radial velocity (RV) and transit techniques currently are the most successful methods for detecting and characterizing properties of exo-planetary systems. The RV surveys have focused on main sequence solar-type stars, with numerous narrow optical lines and low activity, to ensure high RV precision. Recently, planet-search programs have been extended to lower and higher mass stars (Endl et al. 2006; Lagrange et al. 2009a) and younger and more evolved systems (Joergens et al. 2006; Johnson et al. 2007). Since the discovery of 51 Peg b (Mayor \& Queloz 1995), more than 300 exo-planets have been identified featuring a broad range of physical (mass) and orbital $(P, e)$ characteristics (Udry $\&$ Santos 2007; Butler at al. 2006). The RV technique also revealed the existence of a so-called brown dwarf desert at small ( $\leq 5$ AU) orbital separations (Grether \& Lineweaver 2006). The bimodal aspect of the secondary mass distribution indicates different formation mechanisms for two populations of substellar companions, brown dwarfs and planets. The transit technique coupled with RV enables determination of the radius and density of giant planets and thus a probe of their internal structure. Moreover, atmospheric constituents can be revealed during primary or secondary eclipse (Swain et al. 2008; Grillmair et al. 2008).

To extend detection and characterization to orbital semimajor axes $\geq 10 \mathrm{AU}$, the deep imaging technique is essential. To access semimajor axes characteristic of the giant planets of our solar system, even at the nearest stars either the Hubble Space Telescope (HST) or a combination of Adaptive Optics (AO) and a large ground-based telescope (Palomar, CFHT, Keck, Gemini, Subaru, VLT) is mandatory. Moreover, deep imaging surveys take advantage of exhaustive work on identification of young $(\leq 100 \mathrm{Myr})$, nearby $(\leq 100 \mathrm{pc})$ stellar associations. Due to their youth and proximity, such stars offer an ideal niche for detection of warm planetary mass companions that are still moderately bright at near-infrared wavelengths. Since the recognition of the TW Hydrae Association (TWA; Kastner et al. 1997; Webb et al 999), more than 200 young, nearby stars have been identified. Many such stars reside in several coeval moving groups (e.g., TWA, $\beta$ Pictoris, Tucana-Horologium, $\eta$ Cha, AB Dor, Columba and Carinae), sharing common kinematics, photometric and spectroscopic properties (see Zuckerman \& Song 2004, hereafter ZS04; Torres et al. 2008, T08). A few young brown dwarf companions have been detected from space, HR $7329 \mathrm{~B}$ and TWA5 B (Lowrance et al. 2000, 1999), and from the ground, GSC 08047-00232B (Chauvin et al. 2005a). Companions down to the planetary mass regime were discovered around the star AB Pic (Chauvin et al. 2005c) and the young brown dwarf 2M1207 (Chauvin et al. 2004, 2005b). Various deep imaging surveys of young, nearby stars have recently been completed using different high contrast imaging techniques such as coronagraphy, differential imaging or $L$-band imaging (see Table 1).
Table 1. Deep imaging surveys of young ( $<100 \mathrm{Myr})$, nearby $(<100 \mathrm{pc})$ stars dedicated to the search for planetary mass companions and published in the literature.

\begin{tabular}{llllll}
\hline \hline Tel/Instr. & $\begin{array}{l}\text { Mode } \\
\& \text { Filter }\end{array}$ & $\begin{array}{l}\text { FoV } \\
(\operatorname{arcsec})\end{array}$ & $\#$ & $\begin{array}{l}\text { Mass } \\
\left(M_{\text {Jup }}\right)\end{array}$ & Ref. \\
\hline 3.6 m/ADONIS & CI, $H-K$ & $13 \times 13$ & 29 & 5 & $(1)$ \\
NTT/Sharp & Sat-DI, $K$ & $11 \times 11$ & 23 & 5 & $(2)$ \\
NTT/Sofi & Sat-DI, $H$ & $13 \times 13$ & 10 & 5 & $(2)$ \\
HST/NICMOS & DI, $H$ & $19 \times 19$ & 45 & 1 & $(3)$ \\
VLT/NaCo & Sat-DI, $H-K$ & $14 \times 14$ & 28 & 5 & $(4)$ \\
VLT/NaCo & SDI, $H$ & $5 \times 5$ & 45 & 1 & $(5)$ \\
VLT/NaCo & DI, $L^{\prime}$ & $28 \times 28$ & 22 & 1 & $(6)$ \\
Gemini/NIRI & ADI, $H$ & $22 \times 22$ & $85^{*}$ & 1 & $(7)$ \\
\hline
\end{tabular}

- References: (1) Chauvin et al. (2003), (2) Neuhäuser et al. (2003), (3) Lowrance et al. (2005), (4) Masciadri et al. (2005), (5) Biller et al. (2007), (6) Kasper et al. (2007), (7) Lafrenière et al. (2007).

$-\left(^{*}\right)$ : For the Gemini/NIRI survey, half stars have age estimates younger than 200 Myr (see Fig. 1, Lafrenière et al. 2007).

The telescope and the instrument, the imaging mode (CI: coronagraphic imaging; Sat-DI; saturated direct imaging; DI direct imaging; SDI: simultaneous differential imaging; ADI: angular differential imaging) and filters, the field of view (FoV) and the number of stars observed (\#) are given. The typical survey sensitivity in terms of planet mass is reported in each reference. A significant number have reported a null-detection of substellar companions. Kasper et al. (2007), Lafrenière et al. (2007) and Nielsen et al. (2008) initiated a statistical analysis to constrain the physical and orbital properties (mass, period, eccentricity distributions) of a giant planet population. Despite some limitations, the approach is attractive and a first step in characterizing the outer portions of exo-planetary systems.

Deep imaging surveys have also been performed on other classes of targets: distant young associations (Taurus, Chamaeleon, Lupus, Upper Sco), nearby intermediate-age (0.11.0 Gyr) stars, very nearby stars and old stars with planets detected by RV. Some substellar companions were detected with masses near the planet/brown dwarf dividing line: DH Tau (Itoh et al. 2005), GQ Lup (Neuhäuser et al. 2005), CHXR 73 (Luhman et al. 2006), HD 230030 (Metchev et al. 2006) and more recently 1RXS J160929.1-210524 (Lafrenière et al. 2008) and CT Cha (Schmidt et al. 2008). Various teams (McCarthy \& Zuckerman 2004; Carson et al. 2005, 2006; Metchev et al. 2008) have discussed an extension of the brown dwarf desert from small to intermediate semimajor axes. Another purpose was to probe the existence and impact of distant massive substellar companions in exoplanetary systems detected by RV (Patience et al. 2002; Luhman \& Jayawardhana 2002; Chauvin et al. 2006; Mugrauer et al. 2007; Eggenberger et al. 2007). Recently, an important breakthrough was achieved with the imaging detection of planetary mass companions HR 8799 bcd (Marois et al. 2008b), Fomalhaut b (Kalas et al. 2008) and the candidate $\beta$ Pic b (Lagrange et al. 2009b). Such discoveries may become much more common following arrival in coming years of a second generation of deep imaging instruments such as Gemini Planet Imager (GPI; Macintosh et al. 2006) and VLT/SPHERE (Dohlen et al. 2006).

In this paper we report results of a deep coronographic imaging survey whose aim was discovery of substellar companions to young, nearby, austral stars. In comparison to previous work (see Table 1), our survey represents one of the largest and deepest obtained so far on this class of targets. This survey, intitiated in November 2000 with the ADONIS/SHARPII instrument 

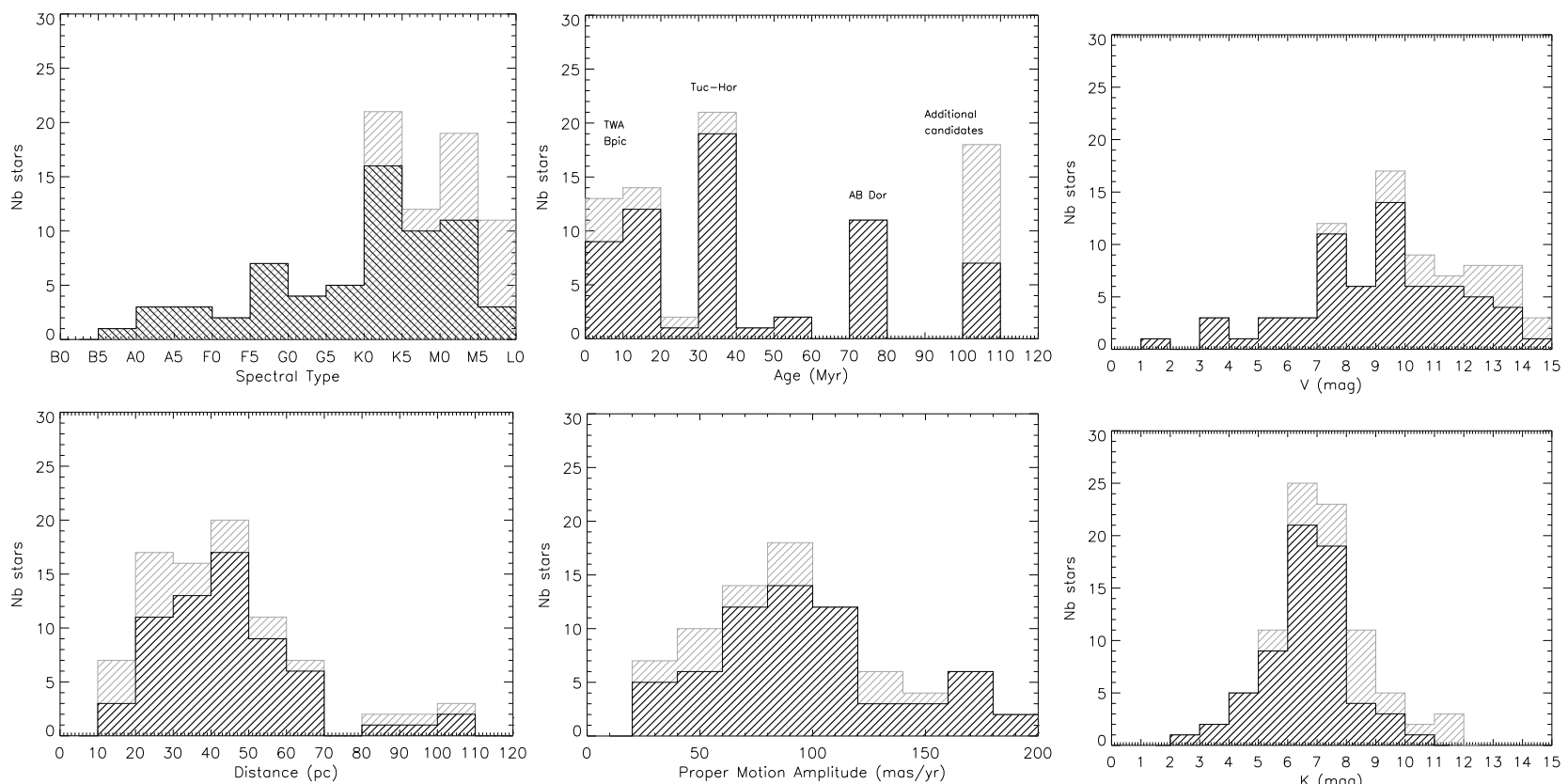

Fig. 1. Histrograms summarizing the main properties of the sample of young, nearby stars observed with NACO at VLT. Top-left: histogram of spectral types for the stars observed in coronagraphic imaging (crossed lines) and in direct imaging (simple lines). Top-middle: histogram of ages for members of known young, nearby associtations (TWA, $\beta$ Pic, Tuc-Hor, AB Dor) and additional young candidates. Top-right: histogram of $V$-band fluxes. The performances of the AO correction with the NACO visible-WFS decreases between $12 \leq V \leq 16$. Bottom-right: histogram of $K$-band fluxes. The coronagraphic mode is not efficient for stars fainter than $K \geq 9-10$.

on a 3.6 m telescope (Chauvin et al. 2003), was then extended with the VLT/NACO instrument between November 2002 and October 2007. In Sect. 2, the sample definition and properties are presented. In Sect. 3, we describe characteristics of the VLT/NACO instrument and the different observing modes that we used. The different observing campaigns, the atmospheric conditions and the observing strategy are detailed in Sect. 4. The dedicated data reduction and analysis to clean the science images, to calibrate our measurments, to derive the relative position and photometry of the detected sources in the NACO field of view and to estimate the detection performances are reported in Sect. 5. We then present the main results of our survey in Sect. 6, including the discovery of new close binary systems and the identification of background contaminants and comoving companions. In Sect. 7, we finally consider the detection sensitivity of our complete survey to statistically constrain the physical and orbital properties of a population of giant planets with 20-150 AU semimajor axes.

\section{Sample selection}

The building up of our target sample relied on a synergy between previous exhaustive work on identification of young, nearby stars and selection criteria (age, distance, binarity and observability) that would optimize the detection of close-in planetary mass companions with NACO at VLT. Youth indicators generally rely on photometry and pre-main sequence isochrones, spectroscopy (especially of lithium and $\mathrm{H}_{\alpha}$ ), and study of X-ray activity and IR excess (see ZS04). Association membership is inferred from coordinates, proper motion, radial velocity and distance estimation. Since the beginning of the present survey, the number of known young, nearby stars more than doubled and newly identified members were regularly included in our target sample. Previously known binaries with 1.0-12.0" separation were excluded to avoid degrading the NACO AO and/or coronagraphic detection performances.

Our initial complete sample was composed of 88 stars; 51 are members of young, nearby comoving groups, 32 are young, nearby stars currently not identified as members of any currently known association and 5 have been reclassified by us as older (>100 Myr) systems. The sample properties are summarized in Tables 2 and 3 and illustrated in Fig. 1.93\% of the selected stars are younger than about $100 \mathrm{Myr}$ and $94 \%$ closer than $100 \mathrm{pc}$. The spectral types cover the sequence from B to $\mathrm{M}$ spectral types with $19 \%$ BAF stars, $48 \%$ GK stars and 33\% M dwarfs. In tables 2 and 3 , in addition to name, coordinates, galactic latitude (b), spectral type, distance and $V$ and $K$ photometry, the observing filter is given. All sources were observed in direct imaging, we have therefore indicated the 65 stars observed in addition in coronagraphy $(\mathrm{CI})$. Finally, the multiplicity status of the primary and the presence of companion candidates (CCs) are also reported. For the multiplicity status we have flagged the following information: binary (B), triple (T) and quadruple (Q); new (N) or known/cataloged (K) multiple system; identified visual (VIS), Hipparcos astrometric (HIP) and spectroscopic (SB) binary system; and a final flag in case of a confirmed physical $(\mathrm{Ph})$ or comoving (Co) system, but nothing if only an optical binary. FS stars are from a paper by Fuhrmeister \& Schmitt (2003).

For stars not in a known moving group (Table 3), based on existing data we employed as many of the techniques for age dating as possible (see, e.g., Sect. 3 in ZS04). The principal diagnostics were lithium abundance, Galactic space motion UVW, and fractional X-ray luminosity (Figs. 3, 6 and 4, respectively in ZS04). With the possible exception of a few of the FS stars (see following paragraph), all Table 3 stars with ages $100 \mathrm{Myr}$ or less have UVW in or near the "good UVW box" in Fig. 6 of ZS04. With the exception of the A-type stars (unknown lithium abundances), all Table 3 stars have lithium abundances (we have measured) consistent with the ages we list and their spectral type 
A\&A 509, A52 (2010)

Table 2. Sample of southern young, nearby stars observed during our VLT/NACO deep imaging survey.

\begin{tabular}{|c|c|c|c|c|c|c|c|c|c|c|c|}
\hline Name & $\begin{array}{l}\alpha \\
{[\mathrm{J} 2000]} \\
\end{array}$ & $\begin{array}{l}\delta \\
\mathrm{J} 2000] \\
\end{array}$ & $\begin{array}{l}b \\
(\operatorname{deg}) \\
\end{array}$ & 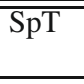 & $\begin{array}{l}d \\
(\mathrm{pc})\end{array}$ & $\begin{array}{l}\begin{array}{l}\text { Age } \\
(\mathrm{Myr})\end{array} \\
\end{array}$ & $\begin{array}{l}V \\
\text { (mag) }\end{array}$ & $\begin{array}{l}K \\
\text { (mag) }\end{array}$ & $\begin{array}{l}\text { Mode } \\
\& \text { Filter }\end{array}$ & $\begin{array}{l}\text { Stellar } \\
\text { multiplicity }\end{array}$ & Note \\
\hline \multicolumn{12}{|c|}{ TWA } \\
\hline TWA22 AB & 101726.9 & -535428 & 2 & M5 & 18 & 8 & 13.2 & 7.69 & $\mathrm{CI}, K_{\mathrm{s}}$ & B (N/VIS/Ph) & $\mathrm{CCs}$ \\
\hline SSSPMJ1102 & 110209.83 & -343035 & 23 & M8 & 65 & 8 & & 11.88 & $K_{\mathrm{s}}$ & & \\
\hline TWA3 AB & 111028.8 & -373204 & 21 & M3 & 42 & 8 & 12.1 & 6.77 & CI, $H$ & B (K/VIS/Co) & \\
\hline Twa14 & 111326.3 & -452343 & 14 & M0 & 63 & 8 & 13.8 & 8.50 & $\mathrm{CI}, K_{\mathrm{s}}$ & & $\mathrm{CCs}$ \\
\hline Twa12 & 112105.6 & -384516 & 21 & M2 & 32 & 8 & 13.6 & 8.05 & $\mathrm{CI}, K_{\mathrm{s}}$ & & $\mathrm{CCs}$ \\
\hline 2M1139 & 113951.1 & -315921 & 28 & M8 & 49 & 8 & & 11.50 & $K_{\mathrm{s}}$ & & \\
\hline HIP57524 & 114724.6 & -495303 & 11 & G5 & 104 & 8 & 9.1 & 7.51 & CI, $H$ & & $\mathrm{CCs}$ \\
\hline Twa23 & 120727.4 & -32470 & 30 & M1 & 37 & 8 & 12.7 & 7.75 & CI, $H$ & & \\
\hline 2M1207 & 120733.4 & -393254 & 23 & M8 & 52 & 8 & & 11.95 & $K_{\mathrm{s}}$ & B (N/VIS/Co) & \\
\hline Twa25 & 121530.7 & -394842 & 22 & M5 & 44 & 8 & 11.4 & 7.31 & $\mathrm{CI}, K_{\mathrm{s}}$ & & \\
\hline HR4796 A & 123601.0 & -395210 & 23 & A0 & 67 & 8 & 5.8 & 5.77 & CI, $H$ & B (K/VIS/Co) & \\
\hline Twa17 & 132045.4 & -461138 & 17 & K5 & 133 & 8 & 12.6 & 9.01 & $\mathrm{CI}, H$ & & $\mathrm{CCs}$ \\
\hline \multicolumn{12}{|c|}{$\beta$ Pictoris } \\
\hline HIP27321 & 054717.0 & -510359 & -31 & A5 & 20 & 12 & 3.9 & 3.53 & $\mathrm{CI}, K_{\mathrm{s}}$ & & \\
\hline V343Nor B & 153856.9 & -574218 & -2 & M4 & 40.0 & 12 & 14.8 & 9.19 & CI, $H$ & $\mathrm{~T}(\mathrm{~K} / \mathrm{VIS}+\mathrm{SB} 2 / \mathrm{Co}+\mathrm{Ph})$ & \\
\hline HD155555 AB & 171725.5 & -665703 & -16 & $\mathrm{~K} 1$ & 31.4 & 12 & 6.9 & 4.70 & CI, $H$ & $\mathrm{~T}(\mathrm{~K} / \mathrm{SB} 2+\mathrm{VIS} / \mathrm{Ph}+\mathrm{Co})$ & $\mathrm{CCs}$ \\
\hline TYC-8742-2065 AB & 174833.7 & -530643 & -13 & K0 & 42 & 12 & 9.0 & 6.78 & $H$ & B (K/SB2 and VIS/Ph) & \\
\hline HIP88399 A & 180303.4 & -513856 & -14 & F5 & 46.9 & 12 & 7.0 & 5.91 & $\mathrm{CI}, K_{\mathrm{s}}$ & B (K/VIS/Co) & $\mathrm{CCs}$ \\
\hline HIP92024 & 184526.9 & -645216 & -24 & A7V & 29.2 & 12 & 4.8 & 4.25 & $\mathrm{CI}, K_{\mathrm{s}}$ & & $\mathrm{CCs}$ \\
\hline CD-641208 AB & 184537.0 & -645146 & -24 & K7 & 29.2 & 12 & 9.5 & 6.10 & CI, $H$ & B (N/VIS) & \\
\hline 0ES1847 & 185044.5 & -314747 & -14 & K5 & 50 & 12 & 10.9 & 7.46 & CI, $H$ & & $\mathrm{CCs}$ \\
\hline HIP92680 & 185305.8 & $\begin{array}{llll}-50 & 1049\end{array}$ & -21 & K0V & 49.6 & 12 & 8.4 & 6.37 & $\mathrm{CI}, K_{\mathrm{s}}$ & & $\mathrm{CCs}$ \\
\hline HIP95270 & 192258.9 & -543216 & -26 & F5 & 50.6 & 12 & 7.0 & 5.91 & $\mathrm{CI}, H$ & & $\mathrm{CCs}$ \\
\hline \multicolumn{12}{|c|}{ Tucana-Horologium } \\
\hline HIP1113 & 001353.01 & -744117 & -42 & G6V & 43.7 & 30 & 8.7 & 6.96 & $\mathrm{CI}, K_{\mathrm{s}}$ & & \\
\hline HIP1481 & 001826.1 & -632838 & -59 & F9V & 41.0 & 30 & 8.0 & 6.15 & $\mathrm{CI}, K_{\mathrm{s}}$ & & $\mathrm{CCs}$ \\
\hline CD-7824 & 004220.2 & -774740 & -40 & K5 & 69 & 30 & 10.4 & 7.53 & CI, $H$ & & \\
\hline HIP3556 & 004528.1 & -513733 & -58 & M1 & 38.5 & 30 & 11.9 & 7.62 & $\mathrm{CI}, K_{\mathrm{s}}$ & & $\mathrm{CCs}$ \\
\hline HIP6485 & 012321.2 & -572850 & -59 & G6 & 49.3 & 30 & 8.5 & 6.85 & $\mathrm{CI}, K_{\mathrm{s}}$ & & $\mathrm{CCs}$ \\
\hline HIP6856 & 012808.6 & -523819 & -64 & K1 & 37.1 & 30 & 9.1 & 6.83 & $\mathrm{CI}, K_{\mathrm{s}}$ & & $\mathrm{CCs}$ \\
\hline HD13246 AB & 020726.1 & -594045 & -55 & F8V & 45.0 & 30 & 7.5 & 6.20 & $\mathrm{CI}, K_{\mathrm{s}}$ & $\mathrm{B}(\mathrm{K} / \mathrm{SB}$ and $\mathrm{VIS} / \mathrm{Ph})$ & \\
\hline GSC08056-00482 & 023651.5 & -520304 & -58 & M3 & 25 & 30 & 12.1 & 7.50 & $\mathrm{CI}, K_{\mathrm{s}}$ & & \\
\hline HIP21632 B & 043845.6 & -270202 & -40 & M3V & 54.7 & 30 & 7.5 & 10.41 & $\mathrm{CI}, K_{\mathrm{s}}{ }^{*}$ & & $\mathrm{CCs}$ \\
\hline HIP30034 & 061912.9 & -580315 & -30 & $\mathrm{~K} 2$ & 45.5 & 30 & 9.1 & 6.98 & CI, $H$ & & $\mathrm{CCs}$ \\
\hline HIP100751 AB & 202538.9 & -564406 & -35 & B7 & 56 & 30 & 1.9 & 2.48 & $\mathrm{CI}, K_{\mathrm{s}}$ & $\mathrm{B}(\mathrm{K} / \mathrm{SB} / \mathrm{Ph})$ & \\
\hline HIP105404 ABC & 212059.8 & -522840 & -44 & K0V & 46.0 & 30 & 8.9 & 6.57 & $\mathrm{CI}, K_{\mathrm{s}}$ & $\mathrm{T}(\mathrm{K} / \mathrm{SB} 3 / \mathrm{Ph})$ & $\mathrm{CC}$ \\
\hline HIP107947 & 215209.7 & -620309 & -44 & F6 & 45 & 30 & 7.2 & 6.03 & $\mathrm{CI}, K_{\mathrm{s}}$ & & $\mathrm{CCs}$ \\
\hline HIP108195 ABC & 215511.4 & -615312 & -45 & F3 & 47 & 30 & 5.9 & 4.91 & $\mathrm{CI}, K_{\mathrm{s}}$ & $\mathrm{T}(\mathrm{K}+\mathrm{N} / \mathrm{VIS} / \mathrm{Ph}+\mathrm{Co})$ & $\mathrm{CCs}$ \\
\hline \multicolumn{12}{|c|}{ AB Dor } \\
\hline HIP5191 A & 010626.1 & -141747 & -76 & K1 & 50 & 70 & 9.5 & 7.34 & CI, $H$ & B (K/VIS/Co) & \\
\hline HIP25283 & 052430.2 & -385811 & -33 & K7 & 18 & 70 & 9.2 & 5.92 & CI, $H$ & B (K/VIS/Co) & \\
\hline $\mathrm{ABD}$ or $\mathrm{BaBb}$ & 052844.3 & -652646 & -33 & M3 & 15 & 70 & 13.0 & 7.34 & CI, $H$ & $\mathrm{Q}(\mathrm{K} / \mathrm{VIS} / \mathrm{Ph})$ & \\
\hline HIP26369 & 053655.1 & -475748 & -32 & K7 & 24 & 70 & 9.8 & 6.61 & CI, $H$ & B (K/VIS/Co) & \\
\hline HIP26373 & 053656.8 & -475753 & -32 & K0 & 24 & 70 & 7.9 & 5.81 & CI, $H$ & B (K/VIS/Co) & \\
\hline HIP30314 & 062230.9 & -601307 & -27 & G0V & 23.5 & 70 & 6.5 & 5.04 & $\mathrm{CI}, K_{\mathrm{s}}$ & B (K/VIS?) & $\mathrm{CCs}$ \\
\hline GSC08894-00426 & 062555.4 & -600329 & -27 & M2 & 22 & 70 & 12.7 & 7.21 & $\mathrm{CI}, K_{\mathrm{s}}$ & & $\mathrm{CCs}$ \\
\hline HIP31878 & 063950.0 & -612842 & -25 & K7 & 21.9 & 70 & 9.7 & 6.50 & $\mathrm{CI}, K_{\mathrm{s}}$ & & \\
\hline HIP76768 AB & 154028.4 & -184145 & 28 & K7 & 43 & 70 & 10.2 & 6.95 & $\mathrm{CI}, K_{\mathrm{s}}$ & B (K/VIS/Co) & $\mathrm{CCs}$ \\
\hline HIP113579 & 230019.2 & -260913 & -65 & G1 & 32 & 70 & 7.5 & 5.94 & $\mathrm{CI}, K_{\mathrm{s}}$ & & $\mathrm{CCs}$ \\
\hline HIP118008 & 235610.7 & -390308 & -77 & $\mathrm{~K} 3$ & 22.1 & 70 & 8.2 & 5.91 & $\mathrm{CI}, H$ & & \\
\hline \multicolumn{12}{|c|}{$\eta$ Cha, Near Cha, Columba and Carina } \\
\hline M0838 & 083851.1 & -791613 & -22 & M5 & 97 & 6 & 16.5 & 10.43 & $\overline{K_{\mathrm{s}}}$ & & \\
\hline HIP58285(TCha) & 115713.7 & -792132 & -16 & F5 & 66.4 & 10 & 11.4 & 6.95 & $\mathrm{CI}, K_{\mathrm{s}}$ & & $\mathrm{CCs}$ \\
\hline GSC08047-00232 A & 015214.6 & -521933 & -62 & K3 & 85 & 30 & 10.9 & 8.41 & $\mathrm{CI}, K_{\mathrm{s}}$ & B (K/VIS/Co) & \\
\hline TYC-9390-0322 AB & 055329.1 & -815653 & -29 & K0 & 54 & 30 & 9.1 & 6.94 & $H$ & B (N/VIS) & \\
\hline
\end{tabular}

$-(*)$ : S13 camera used in this case.

(as per Fig. 3 in ZS04). With the exception of the A-type stars, $\mathrm{X}$-ray fluxes are consistent with Fig. 4 in ZS04 for the indicated ages. Age uncertainties for non-FS stars in Table 3 are typically $50 \%$ of the tabulated age (i.e., $30 \pm 15 \mathrm{Myr}, 100 \pm 50 \mathrm{Myr}$ ). The ages of the two A-type stars are based on UVW and location on a young star HR diagram.
When their radial velocity is known (based on our echelle spectra) then the FS stars usually have a "good UVW". In all cases they are strong X-ray emitters and also have $\mathrm{H}$ alpha in emission, usually strongly. Lithium is usually not detected in the FS stars, or occasionally weakly. Because the data sets for these stars are sometimes incomplete (e.g., radial velocity not 
Table 3. Sample of southern young, nearby stars observed.

\begin{tabular}{|c|c|c|c|c|c|c|c|c|c|c|c|}
\hline Name & $\begin{array}{l}\alpha \\
{[\mathrm{J} 2000]}\end{array}$ & $\begin{array}{l}\delta \\
{[\mathrm{J} 2000]}\end{array}$ & $\begin{array}{l}b \\
\text { (deg) }\end{array}$ & SpT & $\begin{array}{l}d \\
(\mathrm{pc})\end{array}$ & $\begin{array}{l}\text { Age } \\
(\mathrm{Myr})\end{array}$ & $\begin{array}{l}V \\
(\mathrm{mag})\end{array}$ & $\begin{array}{l}K \\
(\mathrm{mag})\end{array}$ & $\begin{array}{l}\text { Mode } \\
\text { \& Filter }\end{array}$ & $\begin{array}{l}\text { Stellar } \\
\text { multiplicity }\end{array}$ & Note \\
\hline \multicolumn{12}{|c|}{ Additional young candidates } \\
\hline BTR99AB & 012317.0 & -794132 & -37 & K0 & 103 & 10 & 10.1 & 7.07 & $\mathrm{CI}, H$ & $\mathrm{~B}(\mathrm{~N} / \mathrm{VIS})$ & \\
\hline CD-53386 AB & 020153.7 & -523453 & -61 & K3 & 120 & 30 & 11.0 & 8.60 & $H$ & $\mathrm{~B}(\mathrm{~N} / \mathrm{VIS})$ & \\
\hline FS75 & 020453.2 & -534616 & -60 & M4 & 30 & 100 & 15.0 & 9.6 & $K_{\mathrm{s}}$ & & \\
\hline FS84 & 022244.2 & -602247 & -53 & M4 & 20 & 100 & 13.7 & 8.2 & $K_{\mathrm{s}}$ & & \\
\hline GSC08862-00019 & 025804.6 & -624115 & -49 & K4 & 138 & 20 & 11.7 & 8.91 & $\mathrm{CI}, K_{\mathrm{s}}$ & & $\mathrm{CCs}$ \\
\hline TYC6461-1120 A & 040003.7 & -290216 & -48 & K0 & 62 & 40 & 9.6 & 7.15 & $\mathrm{CI}, K_{\mathrm{s}}$ & B (N/VIS/Co) & $\mathrm{CCs}$ \\
\hline HIP 28474 AB & 060041.3 & -445350 & -27 & G8 & 53.7 & 100 & 9.1 & 7.32 & CI, $H$ & $\mathrm{~B}(\mathrm{~N} / \mathrm{VIS})$ & \\
\hline FS388 ABC & 064345.3 & -642439 & -25 & M4 & 22 & 100 & 14.0 & 8.4 & $K_{\mathrm{s}}$ & $\mathrm{T}(\mathrm{N} / \mathrm{VIS})$ & \\
\hline FS465 AB & 081739.4 & -824330 & -24 & M4 & 10 & 100 & 12.6 & 6.6 & $K_{\mathrm{s}}$ & $\mathrm{B}(\mathrm{N} / \mathrm{VIS})$ & \\
\hline HIP 41307 & 082539.6 & -035423 & 18 & A0 & 38 & 100 & 3.9 & 4.08 & $\mathrm{CI}, K_{\mathrm{s}}$ & & \\
\hline FS485 & 084722.6 & -495957 & -4 & M2 & 33 & 100 & 12.0 & 7.71 & $K_{\mathrm{s}}$ & & \\
\hline FS488 AB & 085402.4 & -305136 & 9 & M5 & 15 & 100 & 13.4 & 8.10 & $K_{\mathrm{s}}$ & B (N/VIS) & \\
\hline HIP 51386 & 102942.2 & +012928 & 47 & F5 & 31.5 & 50 & 6.9 & 5.52 & $\mathrm{CI}, K_{\mathrm{s}}$ & & $\mathrm{CCs}$ \\
\hline FS588 & 112006.1 & -102947 & 46 & M3 & 20 & 100 & 12.1 & 7.0 & $K_{\mathrm{s}}$ & & \\
\hline HIP 59315 & 121006.4 & -491050 & 13 & G5 & 37.8 & 100 & 8.2 & 6.50 & CI, $H$ & & $\mathrm{CCs}$ \\
\hline CD-497027 & 122155.6 & -494612 & 13 & K0 & 89 & 20 & 10.1 & 8.01 & $K_{\mathrm{s}}$ & & \\
\hline HIP 61468 & 123545.5 & -410119 & 21 & A7 & 34.6 & 100 & 5.1 & 4.57 & CI, $H$ & & \\
\hline TYC-8992-0605 & 123638.9 & -634443 & 0 & K3 & 50 & 10 & 9.9 & 7.37 & CI, $H$ & & $\mathrm{CCs}$ \\
\hline TYC-09012-1005 & 134442.6 & -634749 & -1 & K5 & 95 & 10 & 11.0 & 7.74 & CI, $H$ & & $\mathrm{CCs}$ \\
\hline TYC-7818-0504 AB & 143013.5 & -435009 & 16 & K5 & 100 & 10 & 10.4 & 7.64 & $H$ & $\mathrm{~B}(\mathrm{~N} / \mathrm{VIS})$ & \\
\hline HIP 74405 & 151223.4 & -751515 & -15 & K0 & 50.2 & 100 & 9.4 & 7.38 & CI, $H$ & & \\
\hline TYC-7846-1538 & 155327.3 & -421602 & 9 & G1 & 48 & 30 & 7.9 & 6.34 & CI, $H$ & & $\mathrm{CCs}$ \\
\hline HIP 80448 ABC & 162517.5 & -490852 & 0 & K1 & 45.5 & 100 & 7.1 & 5.70 & $H$ & $\mathrm{~T}(\mathrm{~K} / \mathrm{SB}+\mathrm{VIS} / \mathrm{Ph}+\mathrm{Co})$ & \\
\hline HIP 84642 AB & 171814.7 & -602727 & -13 & K0 & 54.6 & 40 & 9.5 & 7.53 & $\mathrm{CI}, K_{\mathrm{s}}$ & $\mathrm{B}(\mathrm{N} / \mathrm{VIS})$ & $\mathrm{CCs}$ \\
\hline FS903 & 173746.5 & -131447 & 9 & K7 & 45 & 100 & 10.2 & 6.835 & $\mathrm{CI}, K_{\mathrm{s}}$ & & $\mathrm{CCs}$ \\
\hline FS979 AB & 183520.8 & -312324 & -11 & M5 & 18 & 100 & 13.1 & 7.8 & $K_{\mathrm{s}}$ & B (N/VIS) & $\mathrm{CCs}$ \\
\hline FS1017 & 191920.2 & -013354 & -6 & M5 & 25 & 100 & 16.6 & 9.667 & $K_{\mathrm{s}}$ & & $\mathrm{CCs}$ \\
\hline FS1035 & 194212.8 & -204548 & -20 & M5 & 20 & 100 & 14.4 & 8.756 & $K_{\mathrm{s}}$ & & $\mathrm{CCs}$ \\
\hline HIP 98495 & 200035.5 & -725437 & -31 & A0 & 33.3 & 50 & 3.9 & 3.80 & CI, $H$ & & \\
\hline HIP 102626 & 204745.0 & -363540 & -38 & K0 & 44.4 & 30 & 9.4 & 6.79 & CI, $H$ & B (K/HIP?) & \\
\hline FS1136 AB & 214906.2 & -641255 & -43 & M5 & 25 & 100 & 15.5 & 9.5 & $\mathrm{CI}, K_{\mathrm{s}}$ & B (N/VIS) & \\
\hline FS1174 & 224408.0 & -541320 & -54 & M4 & 30 & 100 & 13.4 & 8.5 & $K_{\mathrm{s}}$ & & $\mathrm{CCs}$ \\
\hline \multicolumn{12}{|c|}{ Reclassified as older systems } \\
\hline HIP 7805 & 014024.1 & -605957 & -55 & $\mathrm{~F} 2$ & 67 & $\geq 100$ & 7.7 & 6.63 & CI, $H$ & & \\
\hline HIP 69562 ABC & 141421.3 & -152121 & 42 & $\mathrm{~K} 5 \mathrm{~V}$ & 26.5 & $\geq 100$ & 10.5 & 6.60 & $K_{\mathrm{s}}$ & $\mathrm{T}(\mathrm{N} / \mathrm{VIS})$ & \\
\hline HIP 76107 & 153236.7 & -522121 & 3 & M0 & 30.6 & $\geq 100$ & 11.0 & 7.60 & $\mathrm{CI}, K_{\mathrm{s}}$ & B (K/HIP?) & $\mathrm{CCs}$ \\
\hline HIP 96334 & 193509.7 & -695832 & -29 & G1V & 35.4 & $\geq 100$ & 7.9 & 6.30 & $\mathrm{CI}, K_{\mathrm{s}}$ & & $\mathrm{CCs}$ \\
\hline HIP $107705 \mathrm{AB}$ & 214905.8 & -720609 & -39 & M0 & 16.1 & 200 & 9.8 & 5.65 & $K_{\mathrm{s}}$ & B (N/VIS) & \\
\hline
\end{tabular}

measured) and because fractional X-ray luminosity and UVW are imprecise measures of age, we have assigned an age of 100 Myr to all observed FS stars. Perhaps a few FS stars have ages older than $100 \mathrm{Myr}$ (FS 588 being the most likely of these). But, similarly, some are likely younger than 100 Myr. By assuming an overall uniform age of $100 \mathrm{Myr}$ for the sample of FS stars, we are probably somewhat overestimating their mean age. The age determination of the ensemble of FS stars is likely to be accurate to within about a factor 2 in general, although the age of some FS stars could well lie outside of this range.

\section{Observations}

\subsection{Telescope and instrument}

$\mathrm{NACO}^{1}$ is the first Adaptive Optics instrument that was mounted at the ESO Paranal Observatory near the end of 2001 (Rousset et al. 2002). NACO provides diffraction limited images in the near infrared (nIR). The observing camera CONICA (Lenzen et al. 2002) is equipped with a $1024 \times 1024$ pixel Aladdin InSb array. NACO offers a Shack-Hartmann visible wavefront sensor

\footnotetext{
${ }^{1}$ http://www.eso.org/instruments/naos/
}

and a nIR wavefront sensor for red cool (M5 or later spectral type) sources. nIR wavefront sensing was used on only $8 \%$ of our sample. Note that in May 2004, the CONICA detector was changed and the latter detector was more efficient thanks to an improved dynamic, a lower readout noise and cleaner arrays. Among NACO's numerous observing modes, only the direct and coronagraphic imaging modes were used. The two occulting masks offered for Lyot-coronagraphy have a diameter of $\oslash=0.7^{\prime \prime}$ and $\oslash=1.4^{\prime \prime}$. According to the atmospheric conditions, we used the broad band filters $H$ and $K_{\mathrm{s}}$, the narrow band filters, NB1.64, NB1.75 and $\mathrm{Br} \gamma^{2}$ and a neutral density filter (providing a transmissivity factor of 0.014 ). In order to correctly sample the NACO PSF (better than Nyquist), the S13 and S27 objectives were used, offering mean plate scales of 13.25 and 27.01 mas per pixel and fields of view of $14^{\prime \prime} \times 14^{\prime \prime}$ and $28^{\prime \prime} \times 28^{\prime \prime}$ respectively.

Our deep imaging survey was initiated during guaranteed time observations shared between different scientific programs and scheduled between November 2002 and September 2003. The survey was extended using open time observations between

\footnotetext{
${ }^{2}$ See filters description: http://www.eso.org/instruments/ naco/inst/filters.html
} 
Table 4. Summary of the different observing campaigns of our survey.

\begin{tabular}{llllll}
\hline \hline ESO Program & Mode & $\begin{array}{l}\text { Start. Night } \\
(\mathrm{UT}-d a t e)\end{array}$ & $\begin{array}{l}\text { Night } \\
(\mathrm{Nb})\end{array}$ & $\begin{array}{l}\text { Loss } \\
(\%)\end{array}$ & $\begin{array}{l}\text { Visits } \\
(\mathrm{Nb})\end{array}$ \\
\hline 070.C-0565(A) & GTO-Vis & $26-11-2002$ & 1 & & 6 \\
070.D-0271(B) & GTO-Vis & $16-03-2003$ & 1.5 & & 8 \\
071.C-0507(A) & GTO-Vis & $07-06-2003$ & 0.5 & 50 & 2 \\
071.C-0462(A) & GTO-Vis & $07-09-2003$ & 0.5 & 50 & 2 \\
072.C-0644(A) & OT-Vis & $05-03-2004$ & 1 & 100 & 0 \\
072.C-0644(B) & OT-Vis & $05-03-2004$ & 1 & 0 & 9 \\
073.C-0469(A) & OT-Vis & $27-04-2004$ & 1 & 0 & 16 \\
073.C-0469(B) & OT-Vis & $25-09-2004$ & 1 & 0 & 12 \\
075.C-0521(A) & OT-Vis & $06-05-2005$ & 1 & 0 & 15 \\
075.C-0521(B) & OT-Vis & $19-08-2005$ & 0.5 & 0 & 10 \\
076.C-0554(A) & OT-Vis & $08-01-2006$ & 1 & 0 & 13 \\
076.C-0554(B) & OT-Vis & $26-02-2006$ & 1 & 30 & 8 \\
078.C-0494(A) & OT-Ser & $2006 / 2007$ & 0.7 & 30 & 7 \\
079.C-0908(A) & OT-Ser & 2007 & 1 & 0 & 10 \\
\hline Total & - & - & 11.7 & & 127 \\
\hline
\end{tabular}

March 2004 and June 2007. The open time observations were shared between classical visitor mode and remote service mode as offered by ESO at the Paranal Observatory. For each campaign, we have reported in Table 4 the ESO programme numbers, the observation type, Guaranteed Time (GTO) or Open Time (OT), if obtained in visitor (Vis) or service (Ser) modes, the starting nights of observation, the number of nights allocated and the time loss. Finally, the number of visits, corresponging to the number of observing sequences executed on new and followup targets, is given.

\subsection{Image quality}

For ground-based telescopes, atmospheric conditions have always been critical to ensure astronomical observations of good quality. Although AO instruments aim at compensating the distorsion induced by atmospheric turbulence, the correction quality (generally measured by the strehl ratio and Full Width Half Maximum ( $F W H M$ ) parameters) is still related to the turbulence speed and strength. For bright targets, the NACO AO system can correct for turbulence with a coherent time $\left(\tau_{0}\right)$ longer than $2 \mathrm{~ms}$. For faster $\left(\tau_{0} \leq 2 \mathrm{~ms}\right)$ turbulence, the system is always late and the image quality and the precision of astrometric and photometric measurements are consequently degraded. During our NACO observing runs, the averaged $\tau_{0}$ was about $5 \mathrm{~ms}$ and larger than $2 \mathrm{~ms} 80 \%$ of the time. The average seeing conditions over all runs was equal to $0.8^{\prime \prime}$ (which happens to be the median seeing value measured in Paranal over the last decade ${ }^{3}$ ). Figure 2 shows the (strehl ratio) performances of the NACO AO system with the visible wavefront sensor as a function of the correlation time of the atmosphere $\tau_{0}$, the seeing and the primary visible magnitude. As expected, the degradation of the performances is seen with a decrease of $\tau_{0}$, the coherent length $\left(r_{0}\right.$, inversely proportional to the seeing) and the primary flux. Still, the results clearly demonstrate the good NACO performances and capabilities over a wide range of observing conditions.

\subsection{Observing strategy}

The VLT/NACO survey was conducted as a continuation of our earlier coronographic survey with the ADONIS/SHARPII instrument at the ESO $3.6 \mathrm{~m}$ telescope at La Silla Observatory

\footnotetext{
${ }_{3}$ http://www.eso.org/gen-fac/pubs/astclim/paranal/ seeing/adaptive-optics/stat fwhm.html
}

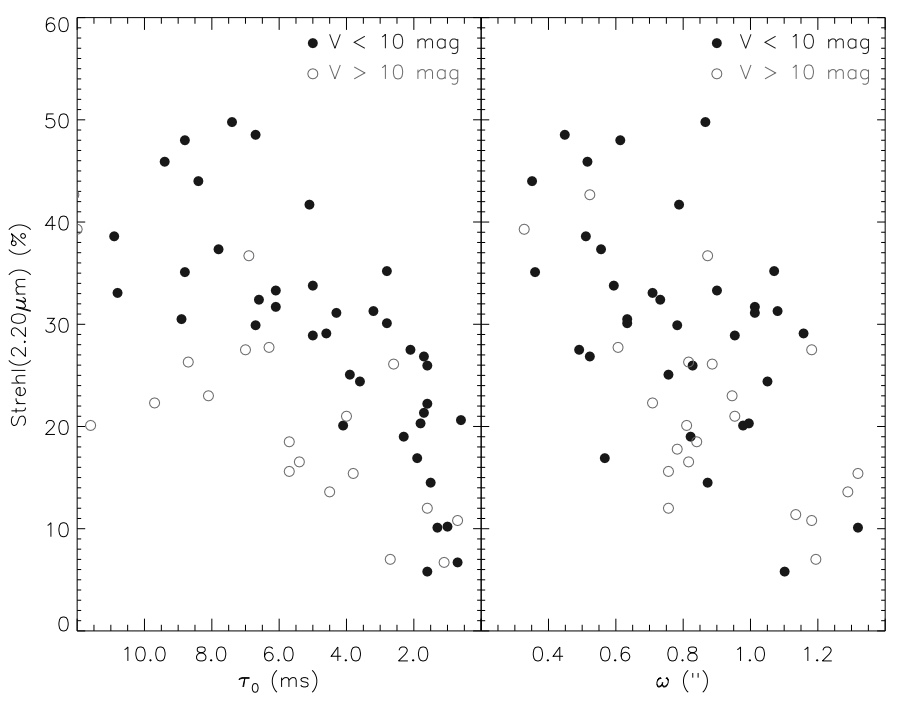

Fig. 2. VLT/NACO adaptive optics system performances. Strehl ratio at $2.20 \mu \mathrm{m}$ as a function of the correlation time $\tau_{0}$ and the seeing $\omega$ of the atmospheric turbulence for two regimes of $V$-band magnitude of the primary star (AO reference target). Only the targets observed with the visible WFS are plotted. Close binaries have also been rejected. The results demonstrate the good behavior of NACO over a wide range of stellar magnitudes and under different turbulent conditions. A clear degradation of the performances is seen for decreasing $\tau_{0}$, increasing $\omega$ and fainter visible $(V \geq 10)$ targets. A clear drop is seen for $\tau_{0}$ faster than $2 \mathrm{~ms}$, the limit of the NACO wavefront sensor sampling frequency.

(Chauvin et al. 2003). A similar observing strategy was adopted to optimize the detection of faint close substellar companions. Most of our stars are relatively bright $\left(K_{\mathrm{s}} \leq 10\right)$ in nIR. To improve our detection performances, we have opted for the use of Lyot coronography. High contrast imaging techniques, such as Lyot and phase mask coronagraphy, $L$-band saturated imaging and simultaneous differential imaging, enable achievement of contrasts of $10^{-5}$ to $10^{-6}$. Their main differences are inherent in the nature of the substellar companions searched and the domain of separations explored. Broad-band nIR Lyot coronagraphy and thermal ( $L^{\prime}$-band or $\left.4 \mu \mathrm{m}\right)$ saturated imaging are among the most sensitive techniques at typical separations between 1.0 to $10.0^{\prime \prime}$. These contrast performances are currently essential to access the planetary mass regime in searches for faint close companions.

To measure precisely positions of faint sources detected in a coronagraphic field relative to the primary star, a dedicated observing block was executed. This block was composed of three successive observing sequences and lasted in total $\sim 45 \mathrm{~min}$ (including pointing). After centering a star behind the coronagraphic mask, a deep coronagraphic observing sequence on source was started. Several exposures of less than one minute each were accumulated to monitor the star centering and the AO correction stability. An effective exposure time of $300 \mathrm{~s}$ was generally spent on target. During the second sequence, a neutral density or a narrow band filter was inserted and the occulting mask and Lyot stop removed. The goal was to precisely measure the star position behind the coronagraphic mask (once corrected for filter shifts). An effective exposure time of $60 \mathrm{~s}$ was spent on source. Counts were adjusted to stay within the $1 \%$ linearity range of the detector. The image is also used to estimate the quality of the AO correction. Finally, the last sequence was the coronagraphic sky. This measure was obtained $\sim 45^{\prime \prime}$ from the star using a jittering pattern of several offset positions to avoid any stellar contaminants in the final median sky. In case of positive detections, whenever possible, the companion 


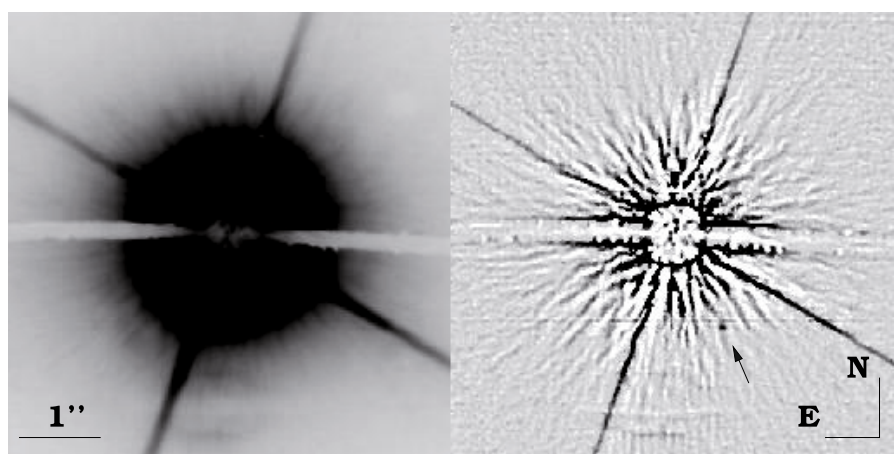

Fig. 3. Left: VLT/NACO corongraphic image of HIP 95270 obtained in $H$-band with the $\mathrm{S} 13$ camera. The small $\left(\varnothing=0.7^{\prime \prime}\right)$ coronagraphic mask was used. Right: coronagraphic image after high-pass filtering. A kernel of $3 \times F W H M$ is used to remove the low spatial frequencies of the coronagraphic PSF wings. A fake $\Delta H=12$ companion has been inserted at $1.2^{\prime \prime}$ from the star to test the detection performances. Minimum and maximum thresholds of the filtered image were divided by a factor 15 to show the fake companion and the PSF residuals.

candidates (CCs) were re-observed to check whether a faint object shared common proper motion with the primary star. Depending on the proper motion of a given star (see Fig. 1), the timespan between successive epochs was about 1-2 years. When comoving companions were identified, images were recorded with addditional nIR filters to directly compare the spectral energy distribution with that predicted by (sub)stellar evolutionary models.

\section{Data reduction and analysis}

\subsection{Cosmetic and image processing}

Classical cosmetic reduction including bad pixels removal, flatfielding, sky substraction and shift-and-add, was made with the Eclipse $^{4}$ reduction software developed by Devillar (1997) for both direct and coronagraphic imaging observations. Median filtering by a kernel of $3 \times 3$ pixels was applied to correct for remaining hot pixels. To remove the central part of the PSF in our reduced coronographic images, two methods were applied. The first method considered different angular sectors uncontaminated by the diffraction spikes and by the coronographic mask support. For each sector, the PSF azimuthal average is calculated, circularised and subtracted from the coronagraphic image. The alternative method was to apply directly a high-pass filter with a kernel of $3 \times F W H M$ (assuming the theoretical $F W H M$ at each observing set-up). As a result, low spatial frequencies, including the coronagraphic PSF wings, were removed from the reduced image. Finally, each resulting image was inspected by eye for identification of any candidate companions. Figure 3 is an illustration of the data processing applied to the coronagraphic images of HIP 95270, in the case of the second method.

\subsection{Astrometric calibration}

The astrometric calibration of high angular resolution images as provided by NACO is not a simple task. As NACO is not a multiconjugated AO system, the diffraction limited images have a small FoV limited by the anisoplanetism angle. Therefore, classical high-precision astrometric techniques over crowded fields of thousands of stars cannot be transposed. In addition, ESO does not currently provide any detector distorsion map. For this

${ }^{4}$ http://www.eso.org/projects/aot/eclipse/
Table 5. Mean plate scale and true north orientation for each observing run.

\begin{tabular}{lllll}
\hline \hline ESO Program & UT Date & Obj. & $\begin{array}{l}\text { Platescale } \\
\text { (mas) }\end{array}$ & $\begin{array}{l}\text { True north } \\
\text { (deg) }\end{array}$ \\
\hline 070.C-0565 & $21-11-2002$ & S13 & $13.24 \pm 0.05$ & $-0.05 \pm 0.10$ \\
& $21-11-2002$ & S27 & $27.01 \pm 0.05$ & $0.08 \pm 0.18$ \\
070.D-0271 & $16-03-2003$ & S13 & $13.21 \pm 0.11$ & $-0.05 \pm 0.10$ \\
$071 . C-0507$ & $29-05-2003$ & S13 & $13.24 \pm 0.05$ & $-0.10 \pm 0.10$ \\
& $03-06-2003$ & S27 & $27.01 \pm 0.05$ & $0.01 \pm 0.19$ \\
071.C-0507 & $07-09-2003$ & S13 & $13.24 \pm 0.05$ & $0.05 \pm 0.10$ \\
072.C-0644 & 05-03-2004 & S13 & $13.24 \pm 0.05$ & $0.04 \pm 0.10$ \\
& $05-03-2004$ & S27 & $27.01 \pm 0.05$ & $-0.18 \pm 0.20$ \\
073.C-0469 & $27-04-2004$ & S27 & $27.01 \pm 0.05$ & $0.08 \pm 0.20$ \\
073.C-0469 & $22-09-2004$ & S13 & $13.25 \pm 0.05$ & $0.20 \pm 0.10$ \\
& $22-09-2004$ & S27 & $27.01 \pm 0.05$ & $0.0 \pm 0.19$ \\
075.C-0521 & $19-08-2005$ & S13 & $13.25 \pm 0.06$ & $-0.02 \pm 0.10$ \\
& $19-08-2005$ & S27 & $27.01 \pm 0.06$ & $-0.07 \pm 0.11$ \\
076.C-0554 & $08-01-2006$ & S13 & $13.25 \pm 0.06$ & $0.18 \pm 0.10$ \\
& $08-01-2006$ & S27 & $27.02 \pm 0.06$ & $0.12 \pm 0.13$ \\
076.C-0554 & $28-02-2006$ & S13 & $13.25 \pm 0.06$ & $0.19 \pm 0.10$ \\
& $28-02-2006$ & S27 & $27.02 \pm 0.05$ & $0.13 \pm 0.14$ \\
$078 . C-0494$ & $24-10-2006$ & S13 & $13.26 \pm 0.07$ & $-0.19 \pm 0.23$ \\
& $23-12-2006$ & S13 & $13.26 \pm 0.08$ & $-0.23 \pm 0.15$ \\
& $22-10-2006$ & S27 & $27.01 \pm 0.03$ & $-0.30 \pm 0.16$ \\
& $25-12-2006$ & S27 & $27.01 \pm 0.04$ & $-0.20 \pm 0.18$ \\
079.C-0908 & $18-07-2007$ & S27 & $27.01 \pm 0.05$ & $-0.06 \pm 0.15$ \\
\hline
\end{tabular}

reason, astrometric calibrators were observed within a week for each observing run (in visitor and service mode) to determine a mean platescale and the true north orientation. Our primary astrometric calibrator was the $\Theta_{1}$ Ori C field observed with HST by McCaughrean \& Stauffer (1994). The same set of stars (TCC058, 057, 054, 034 and 026) were observed with the same observing set-up ( $K_{\mathrm{S}}$ with $\mathrm{S} 27$ and $H$ with $\left.\mathrm{S} 13\right)$ to avoid introduction of systematic errors. When not observable, we used as secondary calibrator the astrometric binary IDS21506S5133 (van Dessel \& Sinachopoulos 1993), yearly recalibrated with the $\Theta_{1}$ Ori $C$ field. The mean orientation of true north and the mean platescale of the S13 and S27 cameras are reported in Table 5.

\subsection{Companion candidate characterization}

For direct imaging, relative photometry and astrometry of visual binaries were obtained using the classical deconvolution algorithm of Véran \& Rigaut (1998). This algorithm is particularly adapted for stellar field analysis. Several PSF references were used to measure the influence of the AO correction. They were selected to optimize a set of observing criteria relative to the target observation (observing time, airmass, spectral type and $V$ or $K$-band flux according to the wavefront sensor).

In coronagraphy, the relative astrometry of the CCs was obtained using a 2D-Gaussian PSF fitting. The deconvolution algorithm of Véran \& Rigaut (1998) and the maximization of the cross-correlation function were applied using the primary star (directly imaged) as PSF reference. The shifts ( $\leq 1$ pixel) induced between direct and coronagraphic images taken with different filters, including neutral density, have been accounted for. For the relative photometry, classical aperture $\left(R_{\mathrm{ap}}=2 \times F W H M\right)$ photometry with residual sky-subtraction and classical deconvolution were used. For faint sources detected at less than $\sim 10 \sigma$, background subtraction becomes more critical and is responsible for larger uncertainties in the deconvolution analysis. Our analysis was then limited to a 2D-Gaussian fitting coupled to aperture photometry to derive the relative astrometry and photometry. 

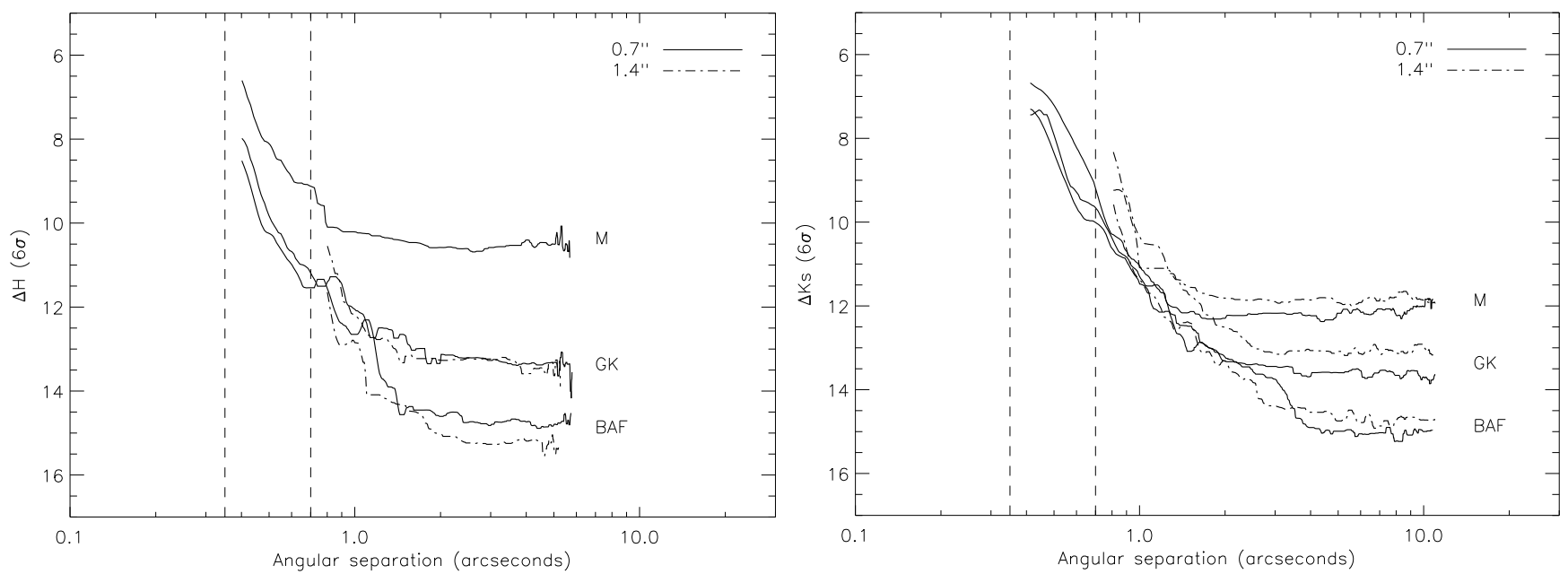

Fig. 4. Left: VLT/NACO coronagraphic detection limits in $H$-band (combined with the $\mathrm{S} 13$ camera). The median detection limits are given for different target spectral types (BAF, GK and M stars) and for the 0.7" (solid line) and 1.4" (dash dotted line) coronagraphic masks. Right: VLT/NACO coronagraphic detection limits in $K_{\mathrm{s}}$-band (combined with the S27 camera). The median detection limits are also given for different target spectral types and coronagraphic masks.

For observations obtained at several epochs, the proper motion and parallactic motion of the primary star were taken into account to investigate the nature of detected faint CCs. The relative positions recorded at different epochs can be compared to the expected evolution of the position measured at the first epoch under the assumption that the $\mathrm{CC}$ is either a stationary background object or a comoving companion (see below). For the range of semi-major axes explored, any orbital motion can be considered of lower order compared with the primary proper and parallactic motions.

\subsection{Detection limits}

The coronagraphic detection limits were obtained using combined direct and coronagraphic images. On the final coronagraphic image, the pixel-to-pixel noise was estimated within a box of $5 \times 5$ pixels sliding from the star to the limit of the NACO field of view. Angular directions free of any spike or coronagraphic support contamination were selected. Additionally, the noise estimation was calculated within rings of increasing radii, a method which is more pessimistic at close angular separation due to the presence of coronagraphic PSF non-axisymmetric residuals. Final detection limits at $6 \sigma$ were obtained after division by the primary star maximum flux and multiplication by a factor taking into account the ratio between the direct imaging and coronagraphic integration times and the difference of filter transmissions and bandwidths. Spectral type correction due to the use of different filters has been simulated and is smaller than $0.04 \mathrm{mag}$. The variation of the image quality (strehl ratio) over the observation remains within $10 \%$ and should not impact our contrast estimation by more than $0.1 \mathrm{mag}$. The median detection limits, using the sliding box method, are reported in Fig. 4. They are given for observations obtained in $H$ - and $K_{\mathrm{s}}$-bands, with the $\oslash=0.7^{\prime \prime}$ and $\oslash=1.4^{\prime \prime}$ coronagraphic masks and for different target spectral types (BAF, GK and M stars) and will be used in the following statistical analysis of the survey.

At large separations $\left(\geq 1.0-2.0^{\prime \prime}\right)$ from the star when limited by detector read-out noise or background noise, the contrast variation with the primary spectral type is actually related to the primary nIR brightness. This is shown in Fig. 5 in the case of $K_{\mathrm{s}}$-band detection limits at $5.0^{\prime \prime}$ as a function of the primary $K_{\mathrm{S}}$

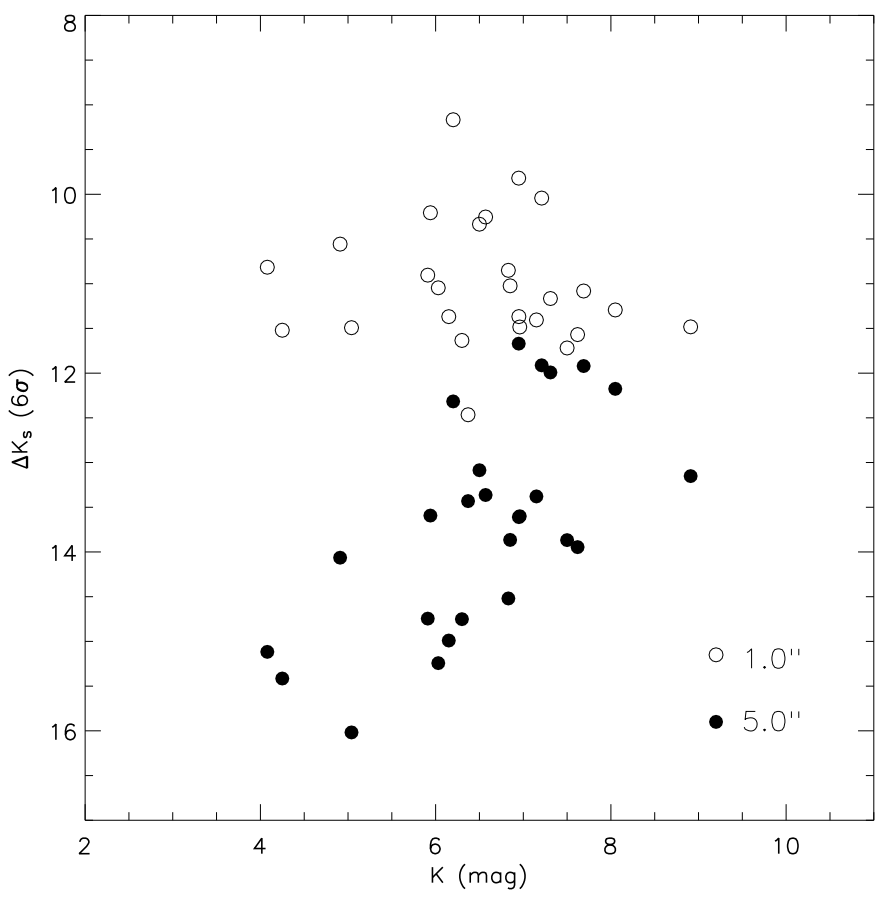

Fig. 5. VLT/NACO coronagraphic detection limits in $K_{\mathrm{s}}$-band as a function of the primary star brightness for two angular separations (1.0" and 5.0"). Two regimes can be seen; one at large separations (shown here at $5.0^{\prime \prime}$ ) where detection is limited by detector read-out noise or background noise. The contrast varies then linearly with the primary $K_{\mathrm{s}}$ apparent magnitude due to the flux normalization; a second regime at smaller separations (shown here at $1.0^{\prime \prime}$ ) when the detection is speckle noise limited. Instrumental quasi-static speckles are expected to dominate random, short-lived atmospheric speckles and the contrast remains relatively constant over a wide range of primary $K_{\mathrm{s}}$ apparent magnitudes.

apparent magnitude. The contrast varies linearly due to the flux normalization. At smaller separations, the situation is more complex as deep AO images are limited by quasi-static speckle noise. Then our detection limits remain constant over a wide range of primary $K_{\mathrm{s}}$ apparent magnitudes. 
All published deep imaging surveys dedicated to planet search (Masciadri et al. 2005; Kasper et al. 2007; Lafrenière et al. 2007; Biller et al. 2007), including this one, derived detection thresholds assuming that residual noise in the final processed image follows a Gaussian intensity distribution. A typical detection threshold at 5 or $6 \sigma$ is then usually assumed over the complete range of angular separations. Whereas the approximation of a Gaussian distribution for the residual noise is valid within the detector read-out noise or background noise regime, careful analysis by Marois et al. (2008a) shows that this is not adequate at small separations when speckle noise limited (typically $\leq 1.0-2.0^{\prime \prime}$ in our survey; see Figs. 4 and 5). In this regime, AO deep images are limited not by random, short-lived atmospheric speckles, but rather by instrumental quasi-static speckles. A non-Gaussian distribution of the residual noise must be taken into account to specify a detection threshold at a given confidence level. Therefore, our current $6 \sigma$ detection threshold at small separations is probably too optimistic. However, the systematic error induced in our sensitivity limits is probably of less significance than uncertainties in planet age and use of uncalibrated planet evolutionary models as described below.

\section{Results}

The main purpose of our survey was detection of brown dwarf and planetary mass companions while employing a deep imaging technique on an optimized sample of nearby stars. Our strategy has been sucessful with the confirmation of a brown dwarf companion to GSC 08047-00232 (Chauvin et al. 2003, 2005a) and discoveries of a planetary mass companion to the young brown dwarf 2MASSW J1207334-393254 (hereafter 2M1207; Chauvin et al. 2004; 2005c) and a companion at the planet/brown dwarf boundary to the young star AB Pic (Chauvin et al. 2005b).

In this section, we detail the three main results of this survey:

1. identification of many background sources along lines of sight close to those of our young, nearby stars. Such identifications are necessary for statistical analysis of our detection limits (see below). These identifications serve in addition as preparation for future deep imaging searches of these stars for exoplanets;

2. discovery of several new close stellar multiple systems, notwithstanding our binary rejection process. Three systems are actually confirmed to be comoving. One is a possible low-mass calibrator for predictions of stellar evolutionary models;

3. review of the status of three previously proposed substellar companions, as confirmed with NACO.

\subsection{Identification of background sources}

Among the complete sample of 88 stars, a total of 65 were observed with coronagraphic imaging. The remaining 23 targets were observed in direct or saturated imaging because the system was resolved as a 1.0-12" visual binary inappropriate for deep coronagraphic imaging, because atmospheric conditions were unstable, or because the system was simply too faint to warrant efficient use of the coronagraphic mode.

Among the 65 stars observed with both direct imaging and coronagraphy, nothing was found around $29(45 \%)$ stars and at least one CC was detected around the $36(55 \%)$ others. A total of $\sim 236$ CCs were detected. To identify their nature, 14 (39\%) systems were observed at two epochs (at least) with VLT and $16(44 \%)$ have combined VLT and HST observations at more than a one year interval (Song et al. 2009, in prep.). Finally, $6(17 \%)$ were observed at only one epoch and require further follow-up observations. The position and photometry of each detected CC relative to its primary star, at each epoch, are given in Tables 8-14. Target name, observing date and set-up are given, as well as the different sources identified with their relative position and relative flux, and their identification status based on follow-up observations. Sources are indicated as undefined (U) were observed at only one epoch, (B) for stationary background contaminants and (C) for confirmed comoving companions. When VLT data are combined with those from other telescopes (HST, USNO, 2MASS), a flag or a reference is reported in the last column.

For multi-epoch observations, to statistically test the probability that the CCs are background objects or comoving companions, a $\chi^{2}$ probability test of $2 \times N_{\text {epochs }}$ degrees of freedom (corresponding to the measurements: separations in the $\Delta \alpha$ and $\Delta \delta$ directions for the number $N_{\text {epochs }}$ of epochs) was applied. This test takes into account the uncertainties in the relative positions measured at each epoch and the uncertainty in the primary proper motion and parallax (or distance). Figure 6 gives an illustration of a $(\Delta \alpha, \Delta \delta)$ diagram that was used to identify a stationary background object near 0ES1847. A status of each CC has been assigned as confirmed companion $\left(\mathrm{C} ; P_{\chi^{2}}<0.1 \%\right)$, background contaminant (B; $P_{\chi^{2}}>99 \%$ ), probably background (PB; $P_{\chi^{2}}>99 \%$, but combining data from two different instruments) and undefined (U). Over the complete coronagraphic sample, $1 \%$ of the CCs detected have been confirmed as comoving companions, $43 \%$ have been identified as probable background contaminants and about $56 \%$ need further follow-up observations. The remaining CCs come mostly from crowded background fields in the field of view of 6 stars observed at one epoch.

Among the 23 stars and brown dwarfs observed only in direct or saturated imaging, several have been resolved as tight multiple systems (see below). 4 stars (FS1174, FS979, FS1017 and FS1035) have at least one substellar CC (see Tables 12, 13 and 14). FS1035 was observed at two successive epochs and the faint source detected at $\sim 5.6^{\prime \prime}$ has been identified as a background object.

\subsection{Close stellar multiple systems}

\subsubsection{New visual binaries}

Our survey was not aimed at detecting new stellar binaries. Known bright equal-mass binaries of 1.0-12.0" separation were rejected from our sample as they degrade the coronagraphic detection performances by limiting dynamical range. A few tight binaries were kept when both components could be placed behind the coronagraphic masks. Despite our binary rejection process, 17 new close visual multiple systems were resolved (see Figs. 7 and 8). They include 13 tight resolved binaries and 4 triple systems. Their relative flux and position are reported in Table 6. Their separations range between $0.1-5.0^{\prime \prime}$ and their $H$ and $K_{\mathrm{s}}$ contrasts between 0.0-4.8 mag. Among them, HIP $108195 \mathrm{ABC}$, HIP $84642 \mathrm{AB}$ and TWA22 AB were observed at different epochs and are confirmed as comoving systems.

\subsubsection{The comoving multiple systems HIP 108195 ABC and HIP 84642 AB}

Close to the Hipparcos double star HIP 108195 AB (F3, 46.5 pc), member of Tuc-Hor, we resolved a faint source at $4.96^{\prime \prime}$ 

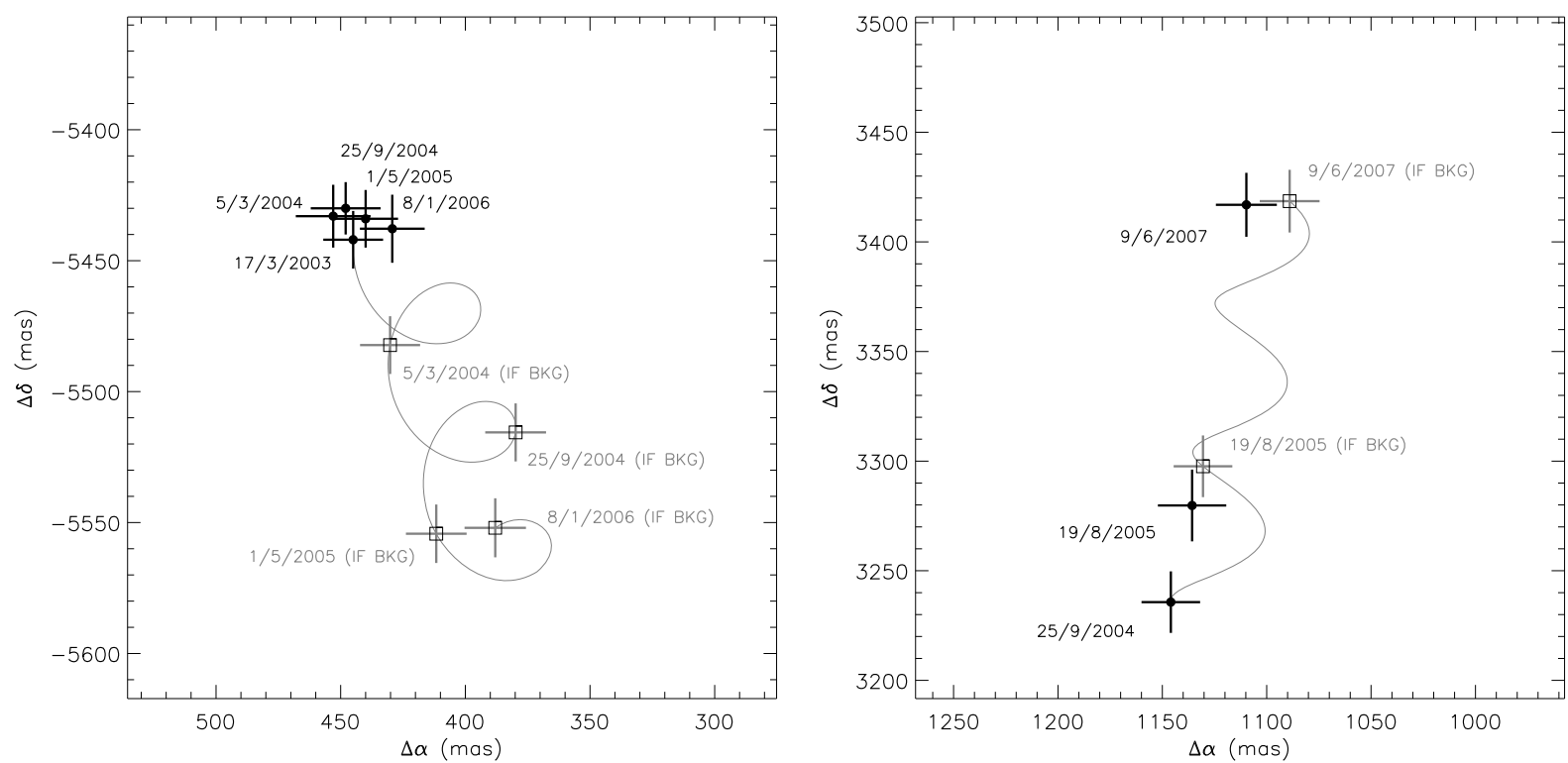

Fig. 6. VLT/NACO Measurements (filled circles with uncertainties) of the offset positions of a comoving companion AB Pic $b$ to the primary star "A" (left) and of a CC relative to 0ES1847 (right). For each diagram, the expected variation of offset positions, if the candidate is a background object, is shown (curved line). The variration is estimated based on the parallactic and proper motions of the primary star, as well as the initial offset position of the $\mathrm{CC}$ from A. The empty squares give the corresponding expected offset positions of a background object for various epochs of observations (with uncertainties). In the case of AB Pic b, the relative positions do not change with time confirming that AB Pic b is comoving. On the contrary, the relative position of the CC to $0 \mathrm{ES} 1847$ varies in time as predicted for a stationary background object. For our sample, astrometric follow-up over 1-2 years enabled a rapid identification of true companions.

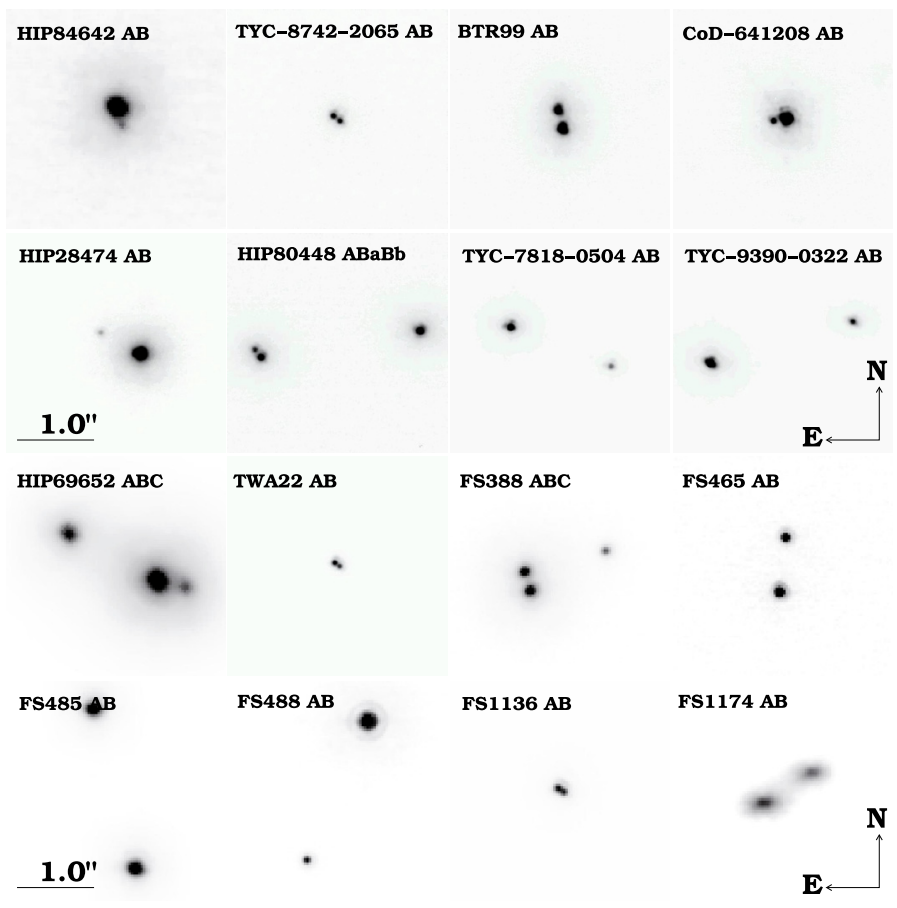

Fig. 7. New visual binaries resolved with NACO at VLT. HIP $108195 \mathrm{AB}, \mathrm{HIP} 84642 \mathrm{AB}$ and TWA22 AB were in addition confirmed as comoving multiple systems. TWA22 AB was monitored for 4 years to constrain the binary orbit and determine its total dynamical mass (see Bonnefoy et al. 2009, accepted).

$\left(\Delta_{\text {proj }}=230 \mathrm{AU}\right.$; i.e. $\left.a \sim 290 \mathrm{AU}\right)$. In addition to a confirmation that HIP $108195 \mathrm{AB}$ is a comoving pair, we found that the fainter source is a third component of this comoving multiple system (Fig. 8). Combined distance, age and apparent photometry are compatible with an M5-M7 dwarf according to PMS

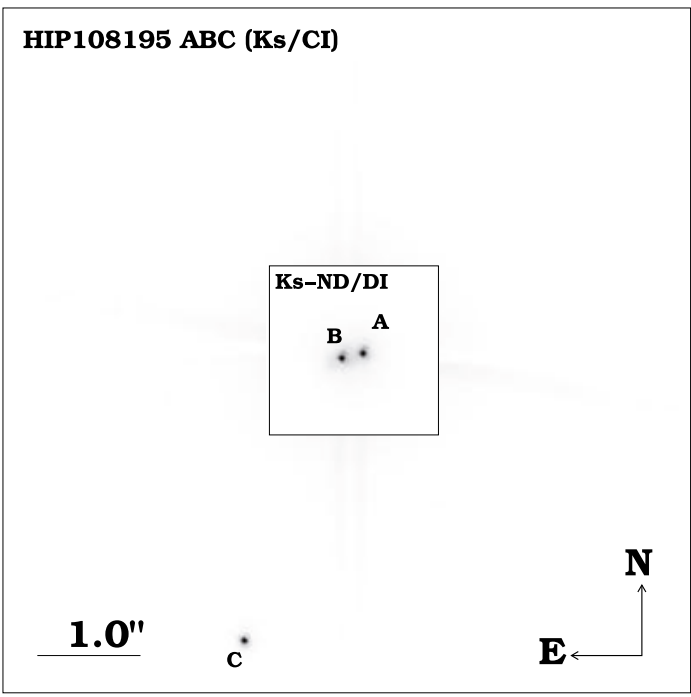

Fig. 8. Composite VLT/NACO $K_{\mathrm{s}}$-band image of the triple comoving system HIP $108195 \mathrm{ABC}$. The inner part shows the direct image (DI) of HIP $108195 \mathrm{AB}$ (attenuated by a factor $\sim 100$ ) using the $K_{\mathrm{s}}$-band with a neutral density filter to avoid saturation. Both components of the astrometric binary cataloged by Hipparcos are resolved. The outer part shows the deeper coronagraphic image $(\mathrm{CI})$ obtained in $K_{\mathrm{s}}$-band with the $\mathrm{C}$ component about 100 times fainter than $\mathrm{A}$ or $\mathrm{B}$.

model predictions (Siess et al. 2000) and places the companion at the stellar/brown dwarf boundary.

HIP 84642 (K0, 58.9 pc) is not reported as a double star in the Hipparcos Visual Double Stars catalog (Dommanget \& Nys 2000), possibly due to the small angular separation and large flux ratio. Based on images from our VLT/NACO programme combined with those from the SACY survey (Huélamo et al. 2009, in prep.), we confirm that the companion shares common proper 
Table 6. Relative positions and $K_{\mathrm{s}}$ and $H$-band contrast of the new binaries resolved by NACO at VLT.

\begin{tabular}{lllll}
\hline \hline Name & UT Date & $\begin{array}{l}\Delta \\
(\mathrm{mas})\end{array}$ & $\begin{array}{l}\text { PA } \\
(\mathrm{deg})\end{array}$ & $\begin{array}{l}\Delta K_{\mathrm{s}}^{*} \\
(\mathrm{mag})\end{array}$ \\
\hline HIP 108195 AB & $19-08-2005$ & $339 \pm 5$ & $102.7 \pm 0.2$ & 0.0 \\
AC & $19-08-2005$ & $4964 \pm 9$ & $158.4 \pm 0.2$ & 4.8 \\
HIP 84642 AB & $05-06-2007$ & $220 \pm 14$ & $191.3 \pm 0.8$ & 2.5 \\
TYC-8742-2065 AB & $27-04-2004$ & $114 \pm 2$ & $232.5 \pm 0.4$ & 0.2 \\
BTR99 AB & $25-09-2004$ & $264 \pm 3$ & $12.8 \pm 0.3$ & 0.5 \\
CoD-641208 AB & $25-09-2004$ & $178 \pm 3$ & $95.3 \pm 0.4$ & 2.3 \\
HIP 80448 BaBb & $27-04-2004$ & $134 \pm 2$ & $37.5 \pm 0.7$ & 1.1 \\
HIP 69652 AB & $26-02-2006$ & $319 \pm 6$ & $256.8 \pm 0.5$ & 1.3 \\
AC & $26-02-2006$ & $1123 \pm 6$ & $61.8 \pm 0.4$ & 0.8 \\
TWA22 AB & $05-03-2004$ & $100 \pm 5$ & $80.2 \pm 0.2$ & 0.4 \\
FS388 AB & $08-01-2006$ & $224 \pm 5$ & $16.4 \pm 0.6$ & 0.3 \\
AC & $08-01-2006$ & $963 \pm 6$ & $297.7 \pm 0.2$ & 1.6 \\
FS465 AB & $08-01-2006$ & $619 \pm 6$ & $353.4 \pm 0.3$ & 0.7 \\
FS488 AB & $08-01-2006$ & $1710 \pm 7$ & $156.6 \pm 0.1$ & 2.8 \\
FS485 AB & $26-02-2006$ & $1862 \pm 7$ & $14.7 \pm 0.1$ & 0.2 \\
FS1136 AB & $19-08-2005$ & $74 \pm 5$ & $122.0 \pm 0.8$ & 0.2 \\
FS1174 AB & $19-08-2005$ & $626 \pm 6$ & $302.7 \pm 0.5$ & 0.4 \\
\hline Name & UT Date & $\Delta$ & PA & $\Delta H$ \\
& & (mas) & (o) & (mag) \\
\hline HIP 28474 AB & $27-04-2004$ & $613 \pm 3$ & $61.7 \pm 0.2$ & 3.8 \\
TYC-7818-0504 AB & $17-03-2003$ & $1463 \pm 6$ & $111.3 \pm 0.2$ & 1.7 \\
TYC-9390-0322 AB & $17-03-2003$ & $2005 \pm 7$ & $286.3 \pm 0.1$ & 1.6 \\
\hline
\end{tabular}

$-\left({ }^{*}\right)$ : Contrast uncertainty is about $0.1 \mathrm{mag}$.

motion with HIP 84642. The companion is likely to be an M4M6 young dwarf based on comparison of photometry to predictions of PMS models. Based on the statistical relation between projected separation and semi-major axis of Couteau (1960), HIP $84642 \mathrm{AB}$ is likely to be a tight $\left(\Delta_{\text {proj }}=14 \mathrm{AU} ; a \sim 18 \mathrm{AU}\right.$; K0-M5) binary with a period of several tens of years.

\subsubsection{The young, tight astrometric binary TWA22 AB}

The tight ( $\sim 100$ mas; $a \sim 1.8$ AU) binary TWA22 AB was observed at several epochs. We aimed at monitoring the system orbit to determine the total dynamical mass using an accurate distance determination $(17.53 \pm 0.21 \mathrm{pc}$, Texeira et al. 2009, submitted). The physical properties (luminosity, effective temperature and surface gravity) of each component were obtained based on near-infrared photometric and spectroscopic observations. By comparing these parameters with evolutionary model predictions, we consider the age and the association membership of the binary. A possible under-estimation of the mass predicted by evolutionary models for young stars close to the substellar boundary is presented in two dedicated papers (Bonnefoy et al. 2009, accepted; Texeira et al. 2009, accepted).

\subsection{Substellar companions}

We review below the latest results about the three substellar companions GSC 08047-00232 B, AB Pic b and 2M1207 b since their initial companionship confirmation. Recent age, distance, astrometric and spectroscopic measurements enable us to refine their predicted physical properties and their origin in regards to other confirmed substellar companions in young, nearby associations.

\subsubsection{GSC 08047-00232 B}

Based on the ADONIS/SHARPII observations of two dozen probable association members of Tuc-Hor, Chauvin et al. (2003) identified a $20 \pm 5 M_{\text {Jup }}$ candidate to GSC 08047-00232 (CoD-52381). This candidate was independently detected by Neuhäuser et al. (2003) with the SHARP instrument at the ESO New Technology Telescope (NTT). Neuhäuser \& Guenther (2004) acquired $H$ - and $K$-band spectra and derived a spectral type M8 \pm 2 , corroborated by Chauvin et al. (2005a). Finally, in the course of our VLT/NACO observations, we confirmed that GSC 08047-00232 B was comoving with A (Chauvin et al. 2005a). Mass, effective temperature, and luminosity of B were determined by comparing its $J H K$ photometry with evolutionary model predictions and the Tuc-Hor age and photometric distance for the system. The results are reported in Table 7 and compared to the complete list of confirmed substellar companions discovered among the young, nearby associations. Tentative spectral types have been determined from nIR spectroscopic observations, whereas masses and effective temperatures are predicted by evolutionary models based on the nIR photometry, the age and the distance to the system. Membership in Tuc-Hor and the assigned age of GSC 08047-00232 AB have been debated for a time. Further studies of loose young associations sharing common kinematical and physical properties recently led Torres et al. (2008) to identify GSC 08047-00232 AB as a highprobability $(80 \%)$ member of the Columba association of age $30 \mathrm{Myr}$, confirming the young age and the brown dwarf status of GSC 08047-00232B.

\subsubsection{AB Picb}

During our survey, a $13 \pm 2 M_{\text {Jup }}$ companion was discovered near the young star AB Pic (Chauvin et al. 2005b). Initially identified by Song et al. (2003) as a member of Tuc-Hor, the membership of AB Pic has been recently discussed by Torres et al. (2008) who attached this star to the young ( 30 Myr) Columba association. Additional astrometric measurements of the relative position of AB Pic b to A firmly confirm the companionship reported by Chauvin et al. (2005b; see Fig. 6, left panel). Based on age, distance and nIR photometry, Chauvin et al. (2005b) derived the physical properties of $\mathrm{AB}$ Pic $\mathrm{b}$ based on evolutionary models (see Table 7). As per the three young substellar companions to TWA5A, HR7329 and GSC 08047-00232, AB Pic b is located at a projected physical separation larger than $80 \mathrm{AU}$. Formation by core accretion of planetesimals seems unlikely because of inappropriate timescales to form planetesimals at such large distances. Gravitational instabilities within a protoplanetary disk (Papaloizou \& Terquem 2001; Rafikov 2005; Boley 2009) or Jeans-mass fragmentation proposed for brown dwarf and stellar formation appear to be more probable pathways to explain the origin of the Table 7 secondaries.

\subsection{3. $2 \mathrm{M} 1207 \mathrm{~b}$}

Among the young candidates of our sample, a small number of very low mass stars and brown dwarfs were selected to take advantage of the unique capability offered by NACO at VLT to sense the wavefront in the IR. Most were observed in direct and saturated imaging. This strategy proved to be successful with the discovery of a planetary mass companion in orbit around the young brown dwarf 2M1207 (Chauvin et al. 2004, 2005c). HST/NICMOS observations independently confirmed this result 
Table 7. Properties of the confirmed comoving substellar companions discovered in the young, nearby associations: TW Hydrae (TWA), $\beta$ Pictoris $(\beta \mathrm{Pic})$, Columba (Col) and Carina (Car).

\begin{tabular}{llllllllll}
\hline \hline Name & Grp & $\begin{array}{l}\text { Age } \\
(\mathrm{Myr})\end{array}$ & $\begin{array}{l}d \\
(\mathrm{pc})\end{array}$ & $\mathrm{SpT}_{\mathrm{A}}$ & $\mathrm{SpT}_{\mathrm{B}}$ & $\begin{array}{l}M_{\mathrm{B}} \\
\left(M_{\mathrm{Jup}}\right)\end{array}$ & $\begin{array}{l}T_{\text {eff }}^{B} \\
(\mathrm{~K})\end{array}$ & $q_{\mathrm{B} / \mathrm{A}}$ & $\begin{array}{l}\Delta_{\text {proj }} \\
(\mathrm{AU})\end{array}$ \\
\hline TWA5 & TWA & 8 & $(45-50)$ & $\mathrm{M} 1.5$ & $\mathrm{M} 8.5$ & $25 \pm 5$ & $2500 \pm 150$ & 0.055 & $93 \pm 10$ \\
HR7329 & $\beta$ Pic & 12 & $48.2_{-1.6}^{+1.8}$ & $\mathrm{~A} 0 \mathrm{~V}$ & $\mathrm{M} 8$ & $25 \pm 5$ & $2550 \pm 150$ & 0.010 & $199 \pm 10$ \\
GSC-08047-00232 & Tuc-Hor/Col & 30 & $(85-95)$ & $\mathrm{K} 3 \mathrm{~V}$ & $\mathrm{M}_{-3}^{+1}$ & $20 \pm 5$ & $2100 \pm 200$ & 0.025 & $295 \pm 30$ \\
AB Pic & Tuc-Hor/Car & 30 & $46.0_{-1.5}^{+1.6}$ & $\mathrm{~K} 2 \mathrm{~V}$ & $\mathrm{L1}_{-1}^{+2}$ & $13 \pm 2$ & $1700 \pm 200$ & 0.015 & $250 \pm 10$ \\
2M1207 & TWA & 8 & $52.4_{-1.1}^{+1.1}$ & $\mathrm{M} 8$ & late-L & $4 \pm 1^{*}$ & $1150 \pm 150^{*}$ & $0.16^{*}$ & $40 \pm 2$ \\
\hline
\end{tabular}

- (*): For 2M1207 b, Mohanty et al. (2007) suggest a higher mass of $8 \pm 2 M_{\mathrm{Jup}}$ and the existence of a circum-secondary edge-on disk to explain their measured effective spectroscopic temperature of $1600 \pm 100 \mathrm{~K}$. (See discussion in Sect. 5.3.3.)

(Song et al. 2006). A low signal-to-noise spectrum in H-band enabled Chauvin et al. (2004) to suggest a mid to late-L dwarf spectral type, supported by its very red nIR colors. Additional low signal-to-noise spectroscopic observations compared with synthetic atmosphere spectra led Mohanty et al. (2007) to suggest an effective spectroscopic temperature of $1600 \pm 100 \mathrm{~K}$ and a higher mass of $8 \pm 2 M_{\mathrm{Jup}}$. To explain the companion under-luminosity, Mohanty et al. (2007) have suggested the existence of a circumsecondary edge-on disk responsible for a gray extinction of $\sim 2.5 \mathrm{mag}$ between 0.9 and $3.8 \mu \mathrm{m}$. However, synthetic atmosphere models clearly encounter difficulties in describing faithfully the late-L to mid-T dwarfs transition $(\sim 1400 \mathrm{~K}$ for field $\mathrm{L} / \mathrm{T}$ dwarfs), corresponding to the process of cloud clearing. Similar difficulties have been encountered by Marois et al. (2008b) to reproduce all photometric data of the three planetary mass companions to HR 8799 that fall also near the edge of the transition from cloudy to cloud-free atmospheres. In the case of $2 \mathrm{M} 1207 \mathrm{~b}$, future spectroscopic or polarimetric observations should help to distinguish between the two scenarios (obscured or nonobscured by a circumstellar disk). Recent precise parallax determinations (Gizis et al. 2007; Ducourant et al. 2008) allowed a reevaluation of the distance and the physical properties of the companion (see Table 7).

\section{Statistical analysis}

\subsection{Context}

Over the past few years, a significant number of deep imaging surveys dedicated to the search for exoplanets around young, nearby stars have appeared (Chauvin et al. 2003; Neuhäuser et al. 2003; Lowrance et al. 2005; Masciadri et al. 2005; Biller et al. 2007; Kasper et al. 2007; Lafreniére et al. 2007). Various instruments and telescopes were used with different imaging techniques (coronagraphy, angular or spectral differential imaging, $L^{\prime}$-band imaging) and observing strategies. None of those published surveys have reported the detection of planetary mass companions that could have formed by a core-accretion model (as expected for a large fraction of planets reported by RV measurements). Several potential planetary mass companions were discovered, but generally at relatively large physical separations or with a small mass-ratio with their primaries, suggesting a formation mechanisms similar to (sub)stellar binaries and stars. Only recently, planet candidates perhaps formed by coreaccretion have been imaged around the A-type stars Fomalhaut (Kalas et al. 2008), HR 8799 (Marois et al. 2008b) and $\beta$ Pictoris (Lagrange et al. 2009b), initiating the study of giant exo-planets at the (mass, distance) scale of our solar system.
Confronted with a null-detection of planets formed by coreaccretion, several groups (Kasper et al. 2007; Lafrenière et al. 2007; Nielsen et al. 2008) have developed statistical analysis tools to exploit the complete deep imaging performances of their surveys. A first approach is to test the consistency of various sets of (mass, eccentricity, semi-major axes) parametric distributions of a planet population in the specific case of a null detection. A reasonable assumption is to extrapolate and normalize planet mass, period and eccentricity distributions using statistical results of RV studies at short periods. Given the detection sensitivity of a survey, the rate of detected simulated planets (over the complete sample) enables derivation of the probability of nondetection of a given planet population associated with a normalized distribution set. Then comparison with a survey nulldetection sample tests directly the statistical significance of each distribution and provides a simple approach for constraining the outer portions of exoplanetary systems.

A second more general approach aims at actually constraining the exoplanet fraction $f$ within the physical separations and masses probed by a survey, in the case of null or of positive detections. Contrary to what was assumed before, $f$ becomes an output of the simulation, that depends on the assumed (mass, period, eccentricity) distributions of the giant planet population. This statistical analysis aims at determining $f$ within a confidence interval as a function of mass and semi-major axis, given a set of individual detection probabilities $p_{j}$ directly linked to the detection limits at each star observed during the survey and the giant planet distributions considered. One can refer to the work of Lafrenière et al. (2007) and Carson et al. (2006), for a general description of the statistical formalism applied for this type of analysis.

For our survey, we will consider the specific case of a null detection of planet formation by core-accretion within a Poisson statistical formalism that leads to a simple analytical solution for the exoplanet fraction upper limit $\left(f_{\max }\right)$. In the following, we will consider both of the above approaches to exploit the full survey detection potential.

\subsection{Simulation description}

The simulation process is similar to the one adopted by Kasper et al. (2007), Lafrenière et al. (2007) and Nielsen et al. (2008). Due to the important spectral type dispersion of our sample, we have included in addition a planet mass dependency on primary mass. The different steps of the simulation process are described below:

1. Our sample to be simulated is composed of 65 stars observed in coronagraphic imaging mode (see Tables 2 and 3). 
G. Chauvin et al.: Deep imaging survey of young, nearby austral stars

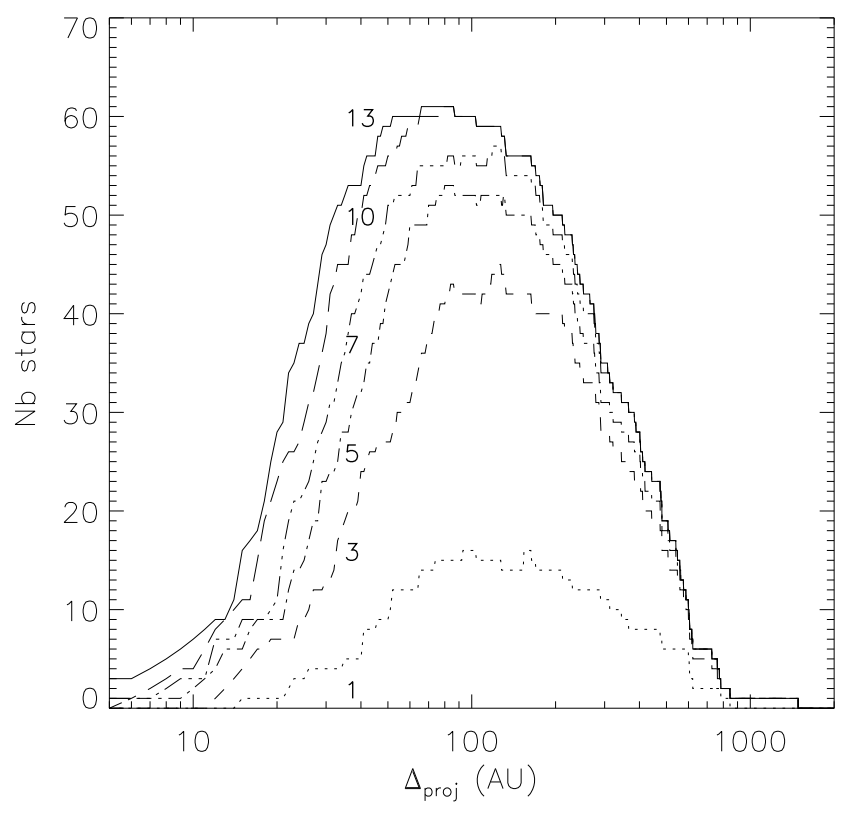

Fig. 9. Histogram of projected physical separations explored, for various planetary masses $\left(1,3,5,7,10\right.$ and 13) $M_{\text {Jup }}$, in the close vicinity of the 65 young, nearby stars observed with NACO at VLT in coronagraphic mode. Contrast performances have been converted into masses based on the nIR photometry, age and distance of the primary stars.

Binaries that could impact the presence of a planet within a range of semi-major axis of $a=$ [5-150] AU were removed. Apparent magnitude, distance, age and mass are the prime simulation parameters.

2. The detection limits were converted to predicted masses using COND03 and DUSTY evolutionary models of Chabrier et al. (2000) and Baraffe et al. (2003). COND03 models are adapted to predict properties of cool $(\leq 1700 \mathrm{~K})$ substellar objects, whereas DUSTY model predictions were considered for hotter temperatures. Based on our $(6 \sigma)$ individual detection limits and target properties (distance, age, $H$ or $K_{\mathrm{s}}$-band magnitude), we derived the range of planet masses and projected physical separation explored around each star of the sample (see histogram in Fig. 9).

3. For the giant planet population, we have considered input distributions based on parametric laws for mass and period extrapolated from RV studies. The eccentricity distribution was chosen to follow the empirical distributions of RV planets. For mass and period, we consider power laws $\mathrm{d} N / \mathrm{d} M_{\mathrm{p}} \sin i \propto\left(M_{\mathrm{p}} \sin i\right)^{\alpha}$ and $\mathrm{d} N / \mathrm{d} P \propto P^{\beta}$ respectively. In addition, a planetary mass distribution scaled as a function of stellar mass $\left(M_{\mathrm{p}} \propto M_{*}^{\gamma}\right)$ was tested.

4. Monte Carlo simulations were run to take into account the the exoplanet distributions and orbital phase. For each run, 10000 values of $M_{\mathrm{p}} \sin i$ and $P$ are randomly generated, following the adopted exoplanet distributions, together with all the other orbital elements, which are supposed to be uniformly distributed. The actual characteristics of each target star (mass, distance from Earth) are taken into account to evaluate the semi-major axis and projected physical separation of the planets.

5. The final step is a comparison with the survey null-detection results and detection sensitivity: either for a derivation of a non-detection probability (thus constraining the statistical significance of various input distributions), or for a derivation of the planet fraction upper limit $\left(f_{\max }\right)$ for a given set
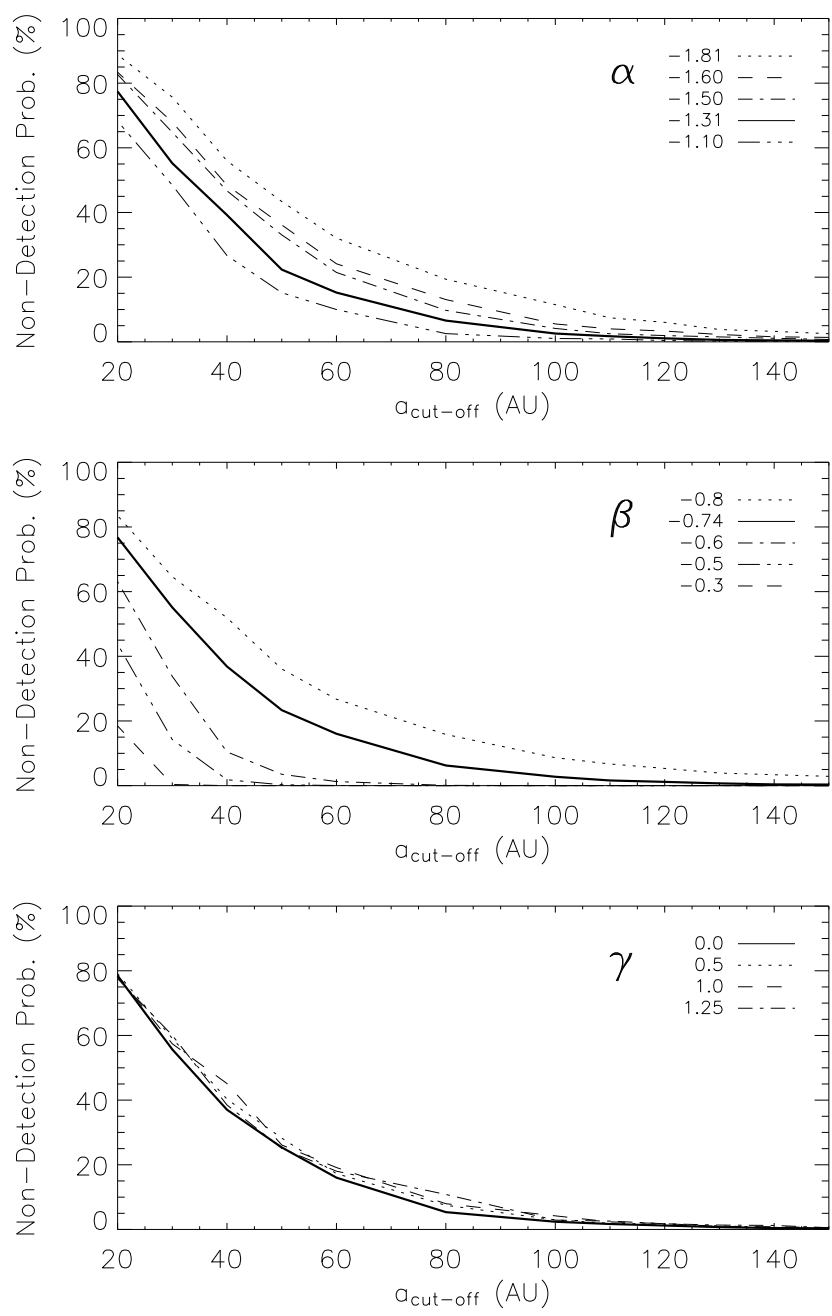

Fig. 10. Non-detection probability for our survey, based on various sets of period and mass distributions as a function of the semi-major axis cut-off of the period distribution. Mass and period distributions are extrapolated and normalized from RV studies. Top: variation of the nondetection probability with $\alpha$ and fixing $\beta=-0.74$ and $\gamma=0.0$. Middle: variation of the non-detection probability with $\beta$ and fixing $\alpha=-1.31$ and $\gamma=0.0$. Bottom: variation with $\gamma$ and fixing $\alpha=-1.31$ and $\beta=-0.74$.

of exoplanet distributions. Dead zones of our coronagraphic images due to the presence of the mask support or a diffraction spike have been considered in our detection sensitivity and simulations.

\subsection{Statistical results}

\subsubsection{Extrapolating radial velocity distributions}

As a starting point, we used the mass and period distributions derived by Cumming et al. (2008) with $\alpha=-1.31$ and $\beta=-0.74$. We considered a giant planet frequency of $8.5 \%$ in the range $0.3-15 M_{\text {Jup }}$ for periods less than 1986 days ( $\leq 3 \mathrm{AU}$ for a $1 M_{\odot}$ host star). The resulting value is consistent with RV studies of Marcy et al. (2005). With several sets of simulations, we explored independently the influence of period, planet mass and primary mass distributions on the non-detection probability determined as a function of the period cut-off. The period cut-off was chosen to correpond to a semi-major axis cut-off between 20 and $150 \mathrm{AU}$. The results, reported in Fig. 10, illuminate the 
impact of the planet mass power law index $\alpha$ with $\beta=-0.74$ and $\gamma=0.0$ (Top), of $\beta$ the period power law index with $\alpha=-1.31$ and $\gamma=0.0$ (Middle), and the evolution implied by a planet mass dependency with the primary mass when $\gamma$ varies and $\alpha=-1.31$ and $\beta=-0.74$ (Bottom). As reference, the Cumming et al. (2008) extrapolated distributions are indicated in thick solid lines in all panels of Fig. 10. As may be seen, the non-detection probability of our survey as a function of the period cut-off is quite sensitive to the variation of $\beta$, the period power law index. In comparison, the influence of $\alpha$ and $\gamma$ remains relatively limited under the current assumptions.

\subsubsection{Exoplanet fraction upper limit}

The probability of planet detection for a survey of $N$ stars is described by a binomial distribution, given a success probability $f p_{j}$ with $f$ the fraction of stars with planets and $p_{j}$ the individual detection probabilities of detecting a planet if present around the star $j$. In our case, we can consider a null detection result and replace each individual $p_{j}$ by $\left\langle p_{j}\right\rangle$ the mean survey detection probability of detecting a planet if present. Assuming that the number of expected detected planets is small compared to the number of stars observed $\left(f\left\langle p_{j}\right\rangle \ll 1\right)$, the binomial distribution can be approximated by a Poisson distribution to derive a simple analytical solution for the exoplanet fraction upper limit $f_{\max }$ for a given level of credibility CL

$f_{\text {max }}=\frac{-\ln (1-\mathrm{CL})}{N\left\langle p_{j}\right\rangle}$.

We consider the mass and period power law indexes from Cumming et al. (2008) $\alpha=-1.31, \beta=-0.74$ and $\gamma=1.25$ for the period and mass distribution of giant planets. For the set of detection limits of our survey, we can then determine $\left\langle p_{j}\right\rangle$, the survey mean probability of detecting a planet if present around each star of our sample. Then, given a confidence level $\mathrm{CL}=0.95$, we obtain $f_{\max }$ as a function of planet mass and semi-major axis. The survey mean detection probability and $f_{\max }$ are reported in Fig. 11. It is important to note that both results depend on the assumed distributions of the giant planet population (mass, period, eccentricity). Similar to other deep imaging surveys, our study begins to constrain the fraction of stars with giant planets to less than $10 \%$ for semi-major axes larger than typically $40 \mathrm{AU}$ for this specific set of period, mass and eccentricity distributions. We also see that we barely constrain the fraction of $1 M_{\text {Jup }}$ planets potentially detectable for $24 \%$ of our targets $\left(67 \%\right.$ for the $3 M_{\text {Jup }}$ planets). Increasing the sample size will enable refinement of the statistical constraints on the upper limits of the fraction of stars with giant planets as a function of their mass and semi-major axis. However, a number of intrinsic limitations (detection threshold, age determination and model calibration) remain that will have to be overcome to draw more robust conclusions. Future work that gathers detection constraints from multiple surveys should help refine our knowledge of the occurence of giant planets with wide orbits (>10 AU) and thus complement RV surveys.

\subsection{Limitations}

Determination of detection thresholds (detailed previously), determination of the ages of young nearby stars, and the use of uncalibrated evolutionary models currently limit the relevancy of all statistical analyses of deep imaging surveys aimed at constraining the population of giant planets.
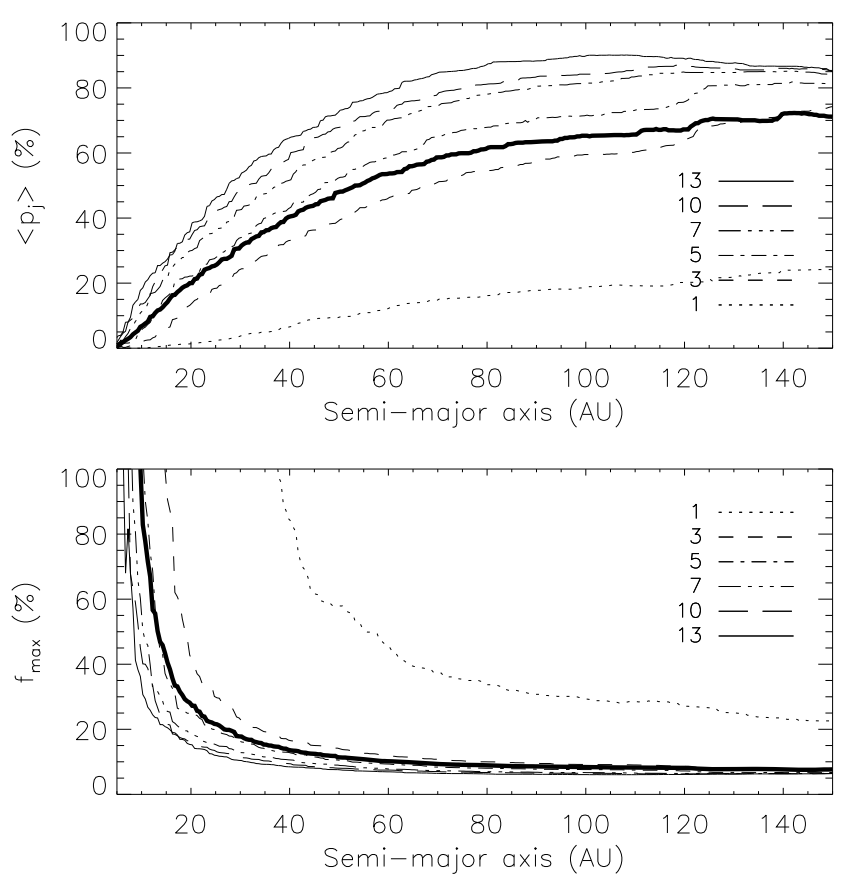

Fig. 11. Top: survey mean detection probability derived as a function of semi-major axis assuming parametric mass and period distributions derived by Cumming et al. (2008), i.e. with $\alpha=-1.31, \beta=-0.74$ and $\gamma=1.25$. The results are reported for individual masses: $1,3,5$, 7, 10 and $13 M_{\text {Jup }}$. The integrated probability for the planetary mass regime is shown with the thick solid line. Bottom: planet fraction upper limit derived as a function of semi-major axis, given the same mass and period distributions.

\subsubsection{Age determination}

Ages of young stars near the Sun are deduced based on photometric, spectroscopic and kinematic studies; various diagnostics are commonly used, depending on the spectral type and age of a given star. Details may be found in ZS04 and T08. In general, the most reliable ages are obtained for stars that can be placed reliably into a moving group or association.

Our sample is composed of 88 stars, including 51 members of known young, nearby associations (TWA, $\beta$ Pic, Tuc-Hor and AB Dor). Ages for the TWA and $\beta$ Pic associations have been reasonably well constrained by various and independent studies (stellar properties and dynamical trace-back): $8_{-3}^{+4}$ Myr (TWA; de la Reza et al. 2006; Barrado y Navasués 2006; Scholz et al. $2007)$ and $12_{-4}^{+8} \operatorname{Myr}(\beta$ Pic, Zuckerman et al. 2001b, Ortega et al. 2004) respectively. Isochrones, lithium depletion and X-ray luminosoties indicate an age for Tuc-Hor of $30 \mathrm{Myr}$ (Zuckerman et al. 2001a). The age of the AB Dor association is in some dispute (see Zuckerman et al. 2004; Luhman et al. 2005; Luhman \& Potter 2006; Lopez-Santiago et al. 2006; Janson et al. 2007; Ortega et al. 2007; Close et al. 2007; Boccaletti et al. 2008; Torres et al. 2008). In our simulations, we have assumed an age of 70 Myr for AB Dor stars.

In our statistical analysis of 65 stars observed in coronagraphic imaging mode, 45 are confirmed members of known associations while 17 are young candidates, currently not identified as members of any kinematic group which makes an age estimate particularly difficult. An excellent example of a young star not known to be a member of the above listed moving groups is HR 8799, identified by Marois et al. (2008b) as orbited by 3 giant planets but with an age uncertainly between 30 and 160 Myr. In our analysis, age is directly used to convert the detection limits 
to mass using evolutionary models. Therefore, age determination remains a main limitation in this work and others to constrain reliably the properties of a putative population of giant planets around young, nearby stars.

\subsubsection{Evolutionary models}

Evolutionary model predictions are commonly used to infer substellar masses from observed luminosities, as we did to convert our survey detection sensitivity into planetary mass limits. For stars and brown dwarfs formed by gravitational collapse and fragmentation, models consider the idealized description of nonaccreting systems contracting at large initial radii. Remaining circumstellar material, accretion and uncertainties related to choice of initial conditions imply that comparison between observations and models are quite uncertain at young $\leq 100 \mathrm{Myr}$ ages (Baraffe et al. 2002). Such a comparison might be rendered even worse should young giant planets form by core-accretion (Marley et al. 2007). Then massive giant planets might be significantly fainter than equal-mass objects formed in isolation via gravitational collapse. However, a critical issue is treatment of the accretion shock through which most of the giant planet mass is processed and which remains highly uncertain. In previous analyses of survey detection sensitivities, only predictions from the Chabrier et al. (2000) and Baraffe et al. (2003) models were used. Adoption of Burrows et al. (2003), assuming the same initial conditions, does not change significantly the results (Nielsen et al. 2008).

\section{Conclusions}

With NACO at the VLT we have conducted a deep adaptive optics imaging survey of 88 nearby stars of the southern hemisphere. Our selection criteria favored youth $(\leq 100 \mathrm{Myr})$ and proximity to Earth $(\leq 100 \mathrm{pc})$ to optimize the detection of planetary mass companions. Known visual binaries were excluded to avoid degrading the NACO AO and/or coronagraphic detection performances. Among our sample, 51 stars are members of young, nearby comoving groups. 32 are young, nearby stars currently not identified as members of any currently known association and 5 have been reclassified as older ( $\geq 100 \mathrm{Myr})$ systems. The spectral types cover the sequence from B to M with $19 \%$ BAF stars, $48 \% \mathrm{GK}$ stars and 33\% $\mathrm{M}$ dwarfs. The separations investigated typically range between $0.1^{\prime \prime}$ and $10^{\prime \prime}$, i.e. between 10 and 500 AU. A sample of 65 stars was observed in deep coronagraphic imaging that enabled sensitivity to star-planet luminosity contrasts as large as $10^{6}$ and, thus, to planetary mass companions down to $1 M_{\text {Jup }}$ (at $24 \%$ of our sample) and $3 M_{\text {Jup }}$ (at $67 \%$ ). We used a standard observing sequence to measure precisely the position and the flux of all detected infrared sources relative to their youthful primary stars. Observations at several epochs enabled us to discriminate comoving companions from background objects. The main results are:

- Discovery of 17 new close (0.1-5.0") multiple systems. HIP $108195 \mathrm{AB}$ and C (F1III-M6), HIP $84642 \mathrm{AB}(a \sim$ 14 AU, K0-M5) and TWA22 AB $(a \sim 1.8$ AU; M6-M6) are confirmed as comoving systems. TWA22 AB, with $80 \%$ of its orbit already resolved, is likely to be a rare astrometric calibrator for testing evolutionary model predictions.

- About 236 faint candidate companions were detected around 36 stars observed in coronagraphic mode. Follow-up observations with VLT or HST for 30 stars enabled us to identify the status of these candidates. $1 \%$ are confirmed as comoving companions, $43 \%$ are identified as probable background contaminants and about $56 \%$ require further follow-up observations (these come mostly from crowded fields near six stars observed at one epoch).

- Confirmation of previously discovered substellar companions around GSC 08047-00232, AB Pic and 2M1207.

- A statistical analysis of the complete set of detection limits enables us to constrain at semi-major axes from 20 to a few $100 \mathrm{AU}$, various mass, period and eccentricity distributions of giant planets extrapolated and normalized from RV surveys. Limits are derived on the occurence of giant planets for a given set of physical and orbital distributions; the survey begins to constrain significantly the population of giant planets with masses $\geq 3 M_{\text {Jup }}$ and with semimajor axes $\geq 40$ AU.

In the first few years following the discovery of the companion to 2M1207 (Chauvin et al. 2004), all planetary mass companions were discovered at relatively wide separations or with small mass ratio with their primaries. However, the recent discoveries of planetary mass objects around the star Fomalhaut (Kalas et al. 2008), HR 8799 (Marois et al. 2008b) and $\beta$ Pictoris (Lagrange et al. 2009b), now open a new era for deep imaging study of giant planets that may have formed like those of our solar system. In the context of current and future deep imaging instruments on the ground (Gemini/NICI, Subaru/HiCIAO, SPHERE, GPI, EPICS) and from space (JWST, TPF/Darwin), the present survey and recent similar ones provide the first constraints on the distribution of masses and semimajor axes of giant planets orbiting at large distances from main sequence stars.

Acknowledgements. We thank the ESO Paranal staff for performing the service mode observations. We also acknowledge partial financial support from the PNPS and Agence National de la Recherche, in France, from INAF through PRIN 2006 "From disk to planetray systems: understanding the origin and demographics of solar and extrasolar planetary systems" and from NASA in the USA. We also would like to thanks France Allard and Isabelle Baraffe for their inputs on evolutionary models and synthetic spectral libraries. Finally, our anonymous referee for her/his detailed and very constructive report.

\section{References}

Baraffe, I., Chabrier, G., Allard, F., \& Hauschildt, P. H. 2002, A\&A 382, 563 Baraffe, I., Chabrier, G., Barman, T. S., Allard, F., \& Hauschildt, P. H. 2003, A\&A, 402, 701

Barrado, Y., \& Navascués, D. 2006, A\&A, 459, 511

Biller, B. A., Close, L. M., Masciadri, E., et al. 2007, ApJS, 173, 143

Boccaletti, A., Chauvin, G., Baudoz, P., \& Beuzit, J.-L. 2008, A\&A, 482, 939 Boley, A. C. 2009, ApJ, 695, L53

Bonnell, I. A., Larson, R. B., \& Zinnecker, H. 2007, Protostars and Planets V, 951, 149

Burgasser, A. J., Kirkpatrick, J. D., Brown, M. E., et al. 1999, ApJ, 522, 65

Burgasser, A. J., Reid, I. N., Siegler, N., et al. 2007, Protostars and Planets V, 951,427

Burrows, A., Sudarsky, D., \& Lunine, J. I. 2003, ApJ, 596, 587

Butler, R. P., Wright, J. T., Marcy, G. W., et al. 2006, ApJ, 646, 505

Carson, J. C., Eikenberry, S. S., Brandl, B. R., Wilson,, J. C., \& Hayward, T. L. 2005, AJ, 130, 1212

Carson, J. C., Eikenberry, S. S., Smith, J. J., \& Cordes, J. M. 2006, AJ, 132, 1146 Chabrier, G., Baraffe, I., Allard, F., \& Hauschildt, P. H. 2000, ApJ, 542, 464

Chauvin, G., Thomson, M., Dumas, C., et al. 2003, A\&A, 404, 157

Chauvin, G., Lagrange, A.-M., Dumas, C., et al. 2004, A\&A, 425, L25

Chauvin, G., Lagrange, A.-M., Lacombe, F., et al. 2005a, A\&A, 430, 1027

Chauvin, G., Lagrange, A.-M., Dumas, C., et al,, 2005b, A\&A, 438, L25

Chauvin, G., Lagrange, A.-M., Zuckerman, B., et al. 2005c, A\&A, 438, L29

Chauvin, G., Lagrange, A.-M., Udry, S., et al. 2006, A\&A, 456, 1165

Close, Laird, M., Thatte, N., Nielsen, E. L., et al. 2007, ApJ, 665, 736

Couteau, P. 1960, J. Obs., 43, 13

Cumming, A., Butler, R. P., Marcy, G. W., et al. 2008, PASP, 120, 531

de la Reza, R., Jilinski, E., \& Ortega, V. G. 2006, AJ, 131, 2609 
Delfosse, X., Tinney, C. G., Forveille, T., et al. 1997, A\&A, 327, 25 Devillar, N. 1997, The messenger, 87

Dohlen, K., Beuzit, J.-L., Feldt, M., et al. 2006, SPIE, 6269, 24 Dommanget, J., \& Nys, O. 2000, A\&A, 363, 991

Ducourant, C., Teixeira, R., Chauvin, G., et al. 2008, A\&A, 477, L1 Eggenberger, A., Udry, S., Chauvin, G., et al. 2007, A\&A, 474, 273 Endl, M., Cochran, W. D., Krster, M., et al. 2006, ApJ, 649, 436 Epchtein, N., de Batz, B., Capoani, L., et al. 1997, Msngr, 87, 27 Fuhrmeister, B., \& Schmitt, J. H. M. M. 2003, A\&A, 403, 247 Gizis, J., Jao, W., Subsavage, J. P., \& Henry, T. J., 2007, ApJ, 669, L45 Goldman, B., Delfosse, X., Forveille, T., et al. 1999, A\&A, 351, L5 Grether, D., \& Lineweaver, C. H., 2006, ApJ, 640, 1051

Grillmair, C. J., Burrows, A., Charbonneau, D., et al. 2008, Nature, 456, 767

Itoh, Y., Hayashi, M., Tamura, M., et al. 2005, ApJ, 620, 984

Janson, M., Brandner, W., Lenzen, R., et al. 2007, A\&A, 462, 615 Joergens, V. 2006, A\&A, 446, 1165

Johnson, J. A., Fischer, D. A., Marcy, G. W., et al. 2007, ApJ, 665, 785

Kalas, P., Graham, J. R., Chiang, E., et al. 2008, Science, 322, 1345

Kastner, J. H., Zuckerman, B., Weintraub, D. A., \& Forveille, T. 1997, Science, 277,67

Kasper, M., Apai, D., Janson, M., \& Brandner, W. 2007, A\&A, 472, 321

Kirkpatrick, J. D., Reid, I. N., Liebert, J., et al. 1999, ApJ, 519, 802

Kirkpatrick, J. D., Reid, I. N., Liebert, J., et al. 2000, ApJ, 120, 447

Lafrenière, D., Doyon, R., Marois, C., et al. 2007, ApJ, 670, 1367

Lafrenière, D., Jayawardhana, R., van Kerkwijk, M. H., et al. 2008, ApJ, 689, 153

Lagrange, A.-M., Desort, M., Galland, F., Udry, S., \& Mayor, M. 2009a, A\&A, 495,335

Lagrange, A.-M., Gratadour, D., Chauvin, G., et al. 2009b, A\&A, 493, L21

Lenzen, R., Hofmann, R., Bizenberger, P., \& Tusche, A. 1998, SPIE, 3354, 606

López-Santiago, J., Montes, D., Crespo-Chacón, I., \& Fernández-Figueroa, M. J. 2006, ApJ, 643, 1160

Lowrance, P. J., McCarthy, C., Becklin, E. E., et al. 1999, ApJ, 512, L69

Lowrance, P. J., Schneider, G., Kirkpatrick, J., et al. 2000, ApJ, 541, L390

Lowrance, P. J., Becklin, E. E., Schneider, G., et al. 2005, AJ, 130, 1845

Luhman, K., \& Jayawardhana, R., 2002, ApJ, 566, 1132

Luhman, K. L., \& Potter, D. 2006, ApJ, 638, 887

Luhman, K. L., Stauffer, J. R., \& Mamajek, E. E. 2005, 628, L69

Luhman, K. L., Wilson, J. C., Brandner, W., et al. 2006, ApJ, 649, 894

Marcy, G., Butler, R. P., Fischer, D., et al. 2005, PThPS, 158, 24

Marley, M. S., Fortney, J. J., Hubickyj, O., Bodenheimer, P., \& Lissauer, J. J. 2007, ApJ, 655, 541

Marois, C., Lafrenière, D., Macintosh, B., \& Doyon, R., 2008a, ApJ, 673, 647

Marois, C., Macintosh, B., Barman, T., et al. 2008b, Science, 322, 1348
Masciadri, E., Mundt, R., Henning, Th., \& Alvarez, C. 2005, ApJ, 625, 1004 Mayor, M., \& Queloz, D. 1995, Nature, 378, 355

McCarthy, C., \& Zuckerman, B. 2004, AJ, 127, 2871

McCaughrean, M. J., \& Stauffer, J. R. 1994, AJ, 108, 1382

Macintosh, B., Troy, M., Doyon, R., et al. 2006, SPIE, 6272, 20

Metchev, S., \& Hillenbrand, L. 2006, ApJ, 651, 1166

Metchev, S., \& Hillenbrand, L. 2008, ApJ, 676, 1281

Mohanty, S., Jayawardhana, R., Huélamo, N., \& Mamajek, E. 2007, ApJ, 657, 1064

Mugrauer, M., Seifahrt, A., \& Neuhäuser, R. 2007, MNRAS, 378, 1328

Nakajima, T., Oppenheimer, B. R., Kulkarni, S. R., et al. 1995, Nature, 378, 463

Neuhäuser, R., \& Guenther, E. W. 2004, A\&A, 420, 647

Neuhäuser, R., Guenther, E. W., Alves, J., et al. 2003, AN, 324, 535

Neuhäuser, R., Guenther, E. W., Wuchterl, G., et al. 2005, A\&A, 435, 13

Nielsen, E. L., Close, L. M., Biller, B. A., Masciadri, E., \& Lenzen, R., 2008, ApJ, 674, 466

Ortega, V. G., de la Reza, R., Jilinski, E., \& Bazzanella, B. 2004, ApJ, 609, 243

Ortega, V. G., Jilinski, E., de La Reza, R., \& Bazzanella, B. 2007, MNRAS, 377 , 441

Papaloizou, J. C. B., \& Terquem, C. 2001, MNRAS, 325, 221

Patience, J., White, R. J., Ghez, A. M., et al. 2002, ApJ, 581, 654 Rafikov, R. R. 2005, ApJ, 621, L69

Rousset, G., Lacombe, F., Puget, P., et al., 2002, SPIE, 4007

Schmidt, T. O. B., Neuhäuser, R., Seifahrt, A., et al. 2008, A\&A, 491, 311

Scholz, A., Coffey, J., Brandeker, A., \& Jayawardhana, R. 2007, ApJ, 662, 1254

Siess, L., Dufour, E., \& Forestini, M. 2000, A\&A, 358, 593

Skrutskie, M. F., Schneider, S. E., Stiening, R., et al. 1997, ASSL, 210, 25

Song, I., Zuckerman, B., \& Bessel, M. S. 2003, ApJ, 599, 342

Song, I., Schneider, G., Zuckerman, B., et al. 2006, ApJ, 26, 282

Swain, M. R., Vasisht, G., \& Tinetti, G. 2008, Nature, 452, 329

Torres, C. A. O., Quast, G. R., Melo, C. H. F., \& Sterzik, M. F. 2008, Handbook of Star Forming Regions, Volume II: The Southern Sky, ASP Monograph Publications, 5, 757 (T08)

Udry, S., \& Santos, N. C. 2007, ARA\&A, 45, 397

van Dessel, E., \& Sinachopoulos, D. 1993, A\&AS, 100, 517

Véran, J. P., \& Rigaut, F. 1998, SPIE, 3353, 426

Webb, R. A., Zuckerman, B., Platais, I., et al. 1999, ApJ, 512, L63

Wilson, J. C., Kirkpatrick, J. D., Gizis, J. E., et al. 2001, AJ, 122, 1989

York, D. G., Adelman, J., Anderson, J. E. Jr, et al. 2000, AJ, 120, 1579

Zuckerman, B., \& Song, I. 2004, ARA\&A, 42, 685 (ZS04)

Zuckerman, B., \& Song, I. 2009, A\&A, 493, 1149

Zuckerman, B., Song, I., \& Webb, R. A. 2001a, ApJ, 559, 388

Zuckerman, B., Song, I., Bessell, M. S., \& Webb, R. A. 2001b, ApJ, 562, 87

Zuckerman, B., Song, I., \& Bessell, M. S., 2004, ApJ, 613, L65 